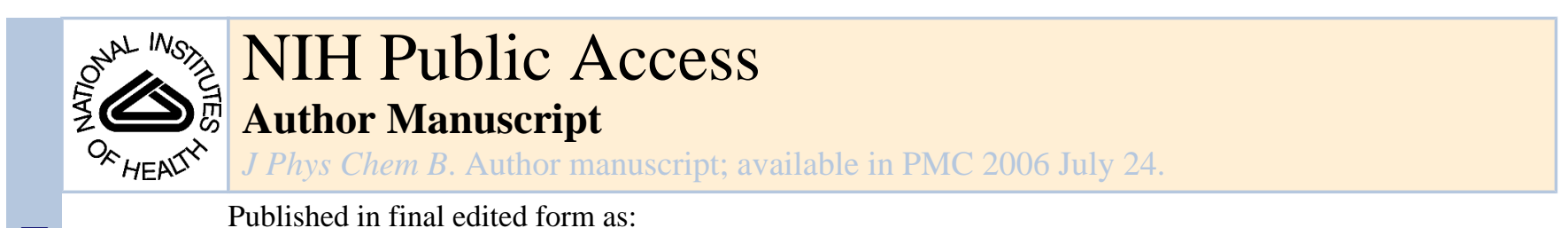

Published in final edited form as:

J Phys Chem B. 2005 November 3; 109(43): 20433-20443.

\title{
Structural Control of the Photodynamics of Boron-Dipyrrin
}

\section{Complexes}

\author{
Hooi Ling Kee ${ }^{1}$, Christine Kirmaier ${ }^{1}$, Lianhe $\mathrm{Yu}^{2}$, Patchanita Thamyongkit ${ }^{2}$, W. Justin \\ Youngblood $^{2}$, Mathew E. Calder ${ }^{2}$, Lavoisier Ramos ${ }^{3}$, Bruce C. Noll ${ }^{4}$, David F. Bocian ${ }^{5}$, W. \\ Robert Scheidt $^{4}$, Robert R. Birge ${ }^{3}$, Jonathan S. Lindsey ${ }^{2}$, and Dewey Holten ${ }^{1}$ \\ 1 Department of Chemistry \\ Washington University \\ St. Louis, MO 63130-4889 \\ Email:holten@wustl.edu \\ 2Department of Chemistry \\ North Carolina State University \\ Raleigh, NC 27695-8204 \\ Email: jlindsey@ncsu.edu \\ 3Departments of Chemistry and of Molecular and Cell Biology \\ University of Connecticut \\ Storrs, CT 06268 \\ Email: rbirge@uconn.edu \\ 4Department of Chemistry \\ University of Notre Dame \\ Notre Dame, IN \\ Email: scheidt.1@nd.edu \\ 5Department of Chemistry \\ University of California Riverside \\ Riverside, CA 92521-0403 \\ Email: dbocian@ucr.edu
}

\section{Abstract}

Boron-dipyrrin chromophores containing a 5-aryl group with or without internal steric hindrance toward aryl rotation have been synthesized and then characterized via X-ray diffraction, static and time-resolved optical spectroscopy, and theory. Compounds with a 5-phenyl or 5-(4-t-butylphenyl) group show low fluorescence yields ( $\sim 0.06)$ and short excited-singlet-state lifetimes ( $\sim 500 \mathrm{ps})$, and decay primarily ( $>90 \%)$ by nonradiative internal conversion to the ground state. In contrast, sterically hindered analogues having an $o$-tolyl or mesityl group at the 5-position exhibit high fluorescence yields $(\sim 0.9)$ and long excited-state lifetimes $(\sim 6 \mathrm{~ns})$. The X-ray structures indicate that the phenyl or 4-tert-butylphenyl ring lies at an angle of $\sim 60^{\circ}$ with respect to the dipyrrin framework whereas the angle is $\sim 80^{\circ}$ for mesityl or $o$-tolyl groups. The calculated potential energy surface for the phenylsubstituted complex indicates that the excited state has a second, lower energy minimum in which the non-hindered aryl ring rotates closer to the mean plane of the dipyrrin, which itself undergoes some distortion. This relaxed, distorted excited-state conformation has low radiative probability as well as a reduced energy gap from the ground state supporting a favorable vibrational overlap factor for nonradiative deactivation. Such a distorted conformation is energetically inaccessible in a 
complex bearing the sterically hindered $o$-tolyl or mesityl group at the 5-position, leading to a high radiative probability involving conformations at or near the initial Franck-Condon form of the excited state. These combined results demonstrate the critical role of aryl-rotation in governing the excitedstate dynamics of this class of widely used dyes.

\section{Introduction}

Boron-dipyrrin dyes were first discovered by Treibs and Kruezer in $1968 .{ }^{1}$ Since then, borondipyrrin dyes have been widely used as markers in life-sciences research. ${ }^{2}$ One limitation to the even broader use of boron-dipyrrin dyes has been the construction of the dipyrrin chromophore using $\beta$-substituted pyrrole units (Chart 1 ), which are available only via quite lengthy synthetic procedures. A decade ago we discovered a simple entrée into 5-substituted dipyrromethanes by reaction of an aldehyde with excess pyrrole at room temperature, ${ }^{3}$ and have since refined this simple procedure. ${ }^{4,5}$ Dolphin showed that such dipyrromethanes can be oxidized to the corresponding free base dipyrrins upon treatment with DDQ or $p$-chloranil. ${ }^{6}$ Complexation of a free base dipyrrin with $\mathrm{BF}_{3} \cdot \mathrm{O}(\mathrm{Et})_{2}$ in the presence of TEA yields the desired boron-dipyrrin complex.

We initially synthesized a boron-dipyrrin dye that incorporated an aryl group at the 5-position and a methyl group at each of the pyrrolic $\alpha$-positions $(\mathbf{1}$, Chart 2$) .{ }^{7,8}$ Such dyes were incorporated in a variety of architectures, including a molecular photonic wire,,, 9 an optoelectronic gate, ${ }^{10}$ and a light-harvesting array. ${ }^{11}$ To our surprise, 5 -substituted borondipyrrin dyes were only weakly fluorescent $\left(\Phi_{\mathrm{f}}=0.058\right.$ for $\left.\mathbf{1}\right)$, in contrast to the intense fluorescence of the analogous $\beta$-substituted dipyrrins first prepared by Treibs and widely used in the life sciences. Time-resolved studies showed that the 5-aryl-substituted dyes exhibited rather short singlet-excited-state lifetime of $\sim 500 \mathrm{ps}$, with an even faster $\sim 15 \mathrm{ps}$ component to the excited-state relaxation dynamics. ${ }^{11} \mathrm{Ab}$ initio calculations indicated that the two kinetic components are associated with two energetically accessible conformers that differ in the rotation of the 5-aryl group and in distortions of the boron-dipyrrin framework. The aryl-ring rotation and accompanying chromophore distortions allow access to an excited-state conformer with low radiative probability and facile nonradiative deactivation to the ground state, thereby limiting the fluorescence yields of the dyes. ${ }^{11}$ The fluorescence properties of an unsubstituted analogue of $\mathbf{1}$, which contained only the 5-phenyl group (2a), also exhibited a low fluorescence quantum yield $\left(\Phi_{\mathrm{f}}=0.053\right) .8$

More recently, we have prepared and characterized a series of bis(dipyrrinato)zinc(II) complexes (Chart 3). ${ }^{12}$ The complex with a 5-phenyl group (3a) exhibits a very short excitedstate lifetime ( $90 \mathrm{ps})$ and a low fluorescence quantum yield (0.006). By contrast, the complex with a 5-mesityl group (3d) exhibits a normal excited-state lifetime ( $\sim 3 \mathrm{~ns})$ and much larger fluorescence quantum yield (0.36). ${ }^{13}$ The photophysical behavior of complex (3b), which contains a 4-tert-butylphenyl group but lacks the 2,6-dimethyl groups, resembles complex 3a, indicating that electronic effects are not the source of the altered excited-state behavior. The profound increase in excited-state lifetime is attributed to steric inhibition of rotation of the 5-mesityl group by the mesityl $o$-methyl substituents. This striking result has prompted us to revisit the boron-dipyrrin complexes with a particular focus on the role of steric effects in controlling the photodynamics of this class of dyes.

In this paper, we describe the synthesis and structural, photophysical, and theoretical characterization of a series of boron complexes of 5-substituted dipyrrin dyes. The substituents include phenyl, 4-tert-butylphenyl, o-tolyl, and mesityl (2a-d, Chart 4). The boron-dipyrrin dyes $\mathbf{2 a - d}$ are more readily synthesized than the corresponding dyes $(\mathbf{4}, \mathbf{5})$ bearing substituents at the pyrrolic $\beta$-positions. We also synthesized and studied five analogs bearing alkyl groups 


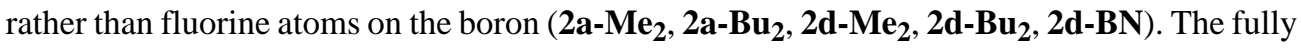
unsubstituted dye (6), although unknown, is a benchmark for theoretical calculations.

\section{Results and Discussion}

Synthesis. The synthesis of the boron-dipyrrin dyes requires access to the corresponding dipyrrin, which is obtained in turn from the dipyrromethane (7). The oxidative conversion of a dipyrromethane to a dipyrrin can be performed with DDQ or $p$-chloranil in THF. 6,12 Dipyrrins $8 \mathbf{a}, 8 \mathbf{b}$, and $8 \mathbf{d}$ have been prepared previously. ${ }^{12}$ The synthesis of dipyrrin $8 \mathbf{c}$ is shown in Scheme 1. Treatment of 5-(o-tolyl)dipyrromethane (7c) $)^{4}$ with DDQ in THF afforded dipyrrin $8 \mathbf{c}$ in $84 \%$ yield.

Boron-dipyrrin complexes such as $\mathbf{2 a}$ were prepared previously from the dipyrromethane (without isolation of the dipyrrin) via a two-step one-flask reaction. ${ }^{4,8,11}$ Here we have employed excess TEA and $\mathrm{BF}_{3} \cdot \mathrm{O}(\mathrm{Et})_{2}$ to complete the reaction (Scheme 2). Thus, treatment of a free base dipyrrin 8 with TEA and $\mathrm{BF}_{3} \cdot \mathrm{O}(\mathrm{Et})_{2}\left(10\right.$ molar equivalents each) in $\mathrm{CH}_{2} \mathrm{Cl}_{2}$ at room temperature for $30 \mathrm{~min}$ afforded the desired boron-dipyrrin complex $\mathbf{2}$ in fair yield (19-68\%). Each boron-dipyrrin complex (2a-d) is less polar than the corresponding starting material (8) or other byproducts and could be easily separated via flash column chromatography. In this manner, boron-dipyrrin complexes 2a-d were obtained.

A series of dialkylboron analogs was prepared in similar fashion. The reaction of dipyrrin 8a

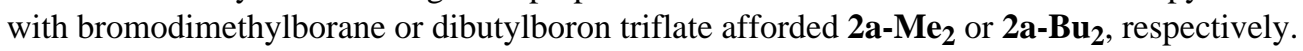
The reaction of 5-mesityldipyrrin (8d) with bromodimethylborane, dibutylboron triflate, or 9-

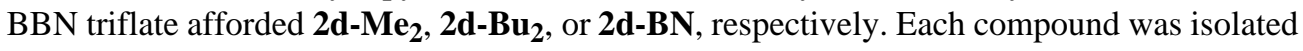
by chromatography on silica and was stable to routine handling.

Structural studies. The results of X-ray diffraction studies of five boron-dipyrrin compounds are described in the Supporting Information. The principal effects of the 5-aryl group and boron substituent can be seen in Table 1 and Figure 1, which give the dihedral angles between various mean planes in the architectures. In all five compounds, the boron-dipyrrin framework, which encompasses the two pyrrole rings and the central six-member ring containing the boron atom, is essentially planar. For the four compounds with fluorines on the boron, the deviation of the pyrrole planes with respect to each other and the central ring is typically only a few degrees.

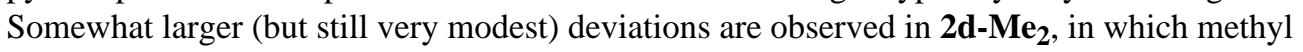
groups replace fluorine atoms on boron. In this case, the dipyrrin framework is slightly puckered, as indicated by the $\sim 15^{\circ}$ dihedral angle between the two pyrrole rings (planes 3 and 4 in Figure 1 and Table 1). For all five compounds, some of the small deviations from coplanarity of the planes in the dipyrrin framework may reflect the effects of crystal packing forces. One example may be the effects of replacement of the 5-phenyl ring in $\mathbf{2 a}$ with the 4tert-butylphenyl ring in $\mathbf{2 b}$.

One of the most pronounced effects of the 5-aryl substituent lies in the dihedral angles between the 5-aryl ring (plane 7) and the planes defining various dipyrrin elements. The angles increase from $\sim 50^{\circ}(\mathbf{2} \mathbf{b}, 4$-tert-butylphenyl $)$ and $\sim 60^{\circ}(\mathbf{2 a}$, phenyl $)$ when no internal hindrance for aryl rotation is present, to $\sim 75^{\circ}(\mathbf{2 d}$, mesityl $)$ and $\sim 85^{\circ}(\mathbf{2 c}, o$-tolyl $)$ when this motion is restricted. Steric hindrance also tends to lengthen the $\mathrm{C} 5$-C10 bond between the dipyrrin and the aryl group, from 1.478(3) for $\mathbf{2 b}$ and 1.4813(11) for 2a to 1.4912(10) for $\mathbf{2 d}$ and 1.4944(17) for 2c. These ground-state structural effects are a harbinger of the distortions involving the planarity of the dipyrrin framework and rotation of the aryl ring that may occur in the excited states of the complexes, as is indicated in the photophysical data and theoretical calculations described below. 
Photophysical characterization. Absorption and Emission Spectra. Figure 2 gives UV/Vis room-temperature electronic ground-state absorption spectra (---) and $\mathrm{S}_{1} \rightarrow \mathrm{S}_{0}$ fluorescence spectra (- - ) of a series of the boron-dipyrrin complexes in cyclohexane (panels A-D). Generally similar spectra are observed in toluene and acetonitrile, and in a solid polyvinyl acetate (PVA) matrix (Supporting Information). Regardless of the presence or nature of a 5aryl ring or the boron substituents, all of the spectra contain a $S_{0} \rightarrow S_{1}$ origin band (490-500 $\mathrm{nm}$ ) and vibronic components spanning $\sim 25 \mathrm{~nm}$ to higher energy. A parallel situation exists in the $\mathrm{S}_{1} \rightarrow \mathrm{S}_{0}$ fluorescence spectra. These vibronic contours are analyzed theoretically below. There is a significant spacing (Stokes shift) of $500-900 \mathrm{~cm}^{-1}$ between the absorption and emission maxima, indicating excited-state conformational changes, solvent reorientation, or both. One of the most notable changes that occurs in the electronic absorption spectrum upon an increase in solvent polarity from cyclohexane (or toluene) to acetonitrile is alteration of the absorption contour in the region between 300 and $400 \mathrm{~nm}$, which includes an increase in intensity of one of the contributing transitions ( $c f$. Figure $2 \mathrm{E}$ ).

Polarized fluorescence and fluorescence-excitation spectra were acquired for $\mathbf{2 a}$ in PVA at room temperature (Supporting Information). The excitation spectra obtained using fluorescence detection at 518,550 , or $650 \mathrm{~nm}$ give a constant polarization ratio of $\sim 0.27$ over the main absorption profile from 400 to $450 \mathrm{~nm}$. These results are consistent with parallel transition dipoles for these absorption and fluorescence features, which can be understood because common $S_{0} \leftrightarrow S_{1}$ transitions are involved. On the other hand, the polarization ratio for the weaker absorption feature(s) near $350 \mathrm{~nm}$ drops below zero, indicating the absorption transition dipole(s) is aligned more perpendicular to that for the $S_{1} \rightarrow S_{0}$ fluorescence. Generally similar polarization results have been obtained previously for $4 .{ }^{13}$

Fluorescence Yields and Lifetimes at Room Temperature. The optical spectra described above indicate that the substituents on the 5-aryl ring of the boron-dipyrrin dyes have only modest impact on the absorption spectra and even less significant effects on the $\mathrm{S}_{1} \rightarrow$ $\mathrm{S}_{0}$ fluorescence profiles. Nonetheless, the nature of the 5-aryl ring has dramatic effects on the fluorescence quantum yield $\left(\Phi_{\mathrm{f}}\right)$ and the lifetime of the $\mathrm{S}_{1}$ excited state $(\tau)$, as shown in Table 2 . These two quantities are related to the fundamental rate constants for $S_{1} \rightarrow S_{0}$ decay via fluorescence $\left(\mathrm{k}_{\mathrm{f}}\right), \mathrm{S}_{1} \rightarrow \mathrm{S}_{0}$ internal conversion $\left(\mathrm{k}_{\mathrm{ic}}\right)$, and $\mathrm{S}_{1} \rightarrow \mathrm{T}_{1}$ intersystem crossing $\left(\mathrm{k}_{\mathrm{isc}}\right)$ via the formulas $\tau=\left(\mathrm{k}_{\mathrm{f}}+\mathrm{kic}+\mathrm{k}_{\text {isc }}\right)^{-1}$ and $\Phi_{\mathrm{f}}=\mathrm{k}_{\mathrm{f}} \div\left(\mathrm{k}_{\mathrm{f}}+\mathrm{kic}+\mathrm{k}_{\mathrm{isc}}\right)=\mathrm{k}_{\mathrm{f}} \bullet \tau$. Table 2 gives estimates for the yields of the two nonradiative decay pathways (internal conversion and intersystem crossing) obtained from the transient absorption data described below. This table also gives estimates for the inverse rate constants (i.e., time constants) for the three $S_{1}$ decay pathways obtained from the excited-state lifetime and corresponding yield via the formula $\left(\mathrm{k}_{\mathrm{i}}\right)^{-1}=\tau \div \Phi_{\mathrm{i}}$. For comparison, Table 2 gives our prior results for toluene solutions of $2 \mathbf{a}$ and $\mathbf{1}$ (Charts 2), ${ }^{11}$ along with fluorescence yield and lifetime data for the boron dipyrrins in PVA (and ethylene glycol). These latter studies were undertaken to determine if motional restrictions imposed by the medium would influence the $\mathrm{S}_{1}$ photophysics at room temperature.

The fluorescence quantum yield found here for phenyl-substituted boron dipyrrin 2a in toluene $\left(\Phi_{\mathrm{f}}=0.062\right)$ is in good agreement with our earlier value $(0.053) .{ }^{11} \mathrm{We}$ also obtained the same excited-state lifetime determined by fluorescence decay $(\tau=0.45 \mathrm{~ns})$. Very similar results are obtained for the 4-tert-butylphenyl analogue $2 \mathbf{b}\left(\Phi_{\mathrm{f}}=0.069, \tau=0.55 \mathrm{~ns}\right)$. In contrast, the introduction of steric constraints on the aryl rings causes bright emission and long excited-state lifetimes. In particular, the $o$-tolyl-substituted complex $2 \mathbf{c}$ in toluene has $\Phi_{\mathrm{f}}=0.93$ and $\tau=5.8$ ns, and the mesityl analogue $\mathbf{2 d}$ has $\Phi_{\mathrm{f}}=0.93$ and $\tau=6.6 \mathrm{~ns}$. Interestingly, relatively strong fluorescence was also observed for $\mathbf{2 d -}-\mathbf{M e}_{2}\left(\Phi_{\mathrm{f}}=0.40\right.$ and $\left.\tau=4.0 \mathrm{~ns}\right)$, which contains methyl groups in place of hydrogen atoms on boron. On the other hand, the fluorescence quantum

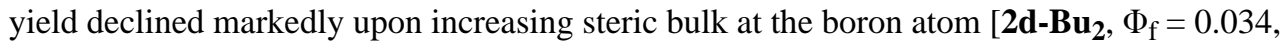
$\left.\tau=3.4 \mathrm{~ns} ; \mathbf{2 d - B N}, \Phi_{\mathrm{f}}=0.004\right]$. Quite low fluorescence yield was observed with the 
dialkylboron analogs of the phenyl-substituted dipyrrin [2-a-Me $\mathbf{2}_{2}, \Phi_{\mathrm{f}}=0.008 ; \mathbf{2} \mathbf{a}-\mathbf{B} \mathbf{u}_{2}, \Phi_{\mathrm{f}}=$ 0.008]. The differences in fluorescence behavior may derive from motions involving the alkyl groups; however, these compounds are somewhat unstable under prolonged illumination and were not studied in detail.In agreement with previous results, ${ }^{14-17}$ intense fluorescence and long excited-state lifetimes are also obtained for $\mathbf{4}$ and $\mathbf{5}$, which contain fluorine atoms on boron, hydrogen or methyl groups (rather than an aryl substituent) at the 5-position along, and methyl groups on the pyrrole rings (Table 2). The fluorescence lifetimes and yields (in toluene) for all of the boron-dipyrrin complexes listed in Table 2 give a radiative rate constant in the range $\mathrm{k}_{\mathrm{f}}=(6-10 \mathrm{~ns})-^{1}$.

These findings indicate that the low emission yields and short excited-state lifetimes found in aryl-substituted complexes bearing no internal steric hindrance derive from a facile $\mathrm{S}_{1}$-excitedstate nonradiative decay channel that is restricted when internal steric constraints are imposed (or in the absence of a 5-aryl ring). As is shown below from the transient absorption data and theoretical results, these low emission yields and short $S_{1}$ excited-state lifetimes result from excited-state conformational changes that drive fast internal conversion to the ground state. In addition to internal steric hindrance, these motions also can be restricted by external forces. For example, Table 2 shows that the fluorescence yield and lifetime essentially double for 2a when the medium is changed from toluene solution $\left(\Phi_{\mathrm{f}}=0.062, \tau=0.45 \mathrm{~ns}\right)$ to the solid PVA matrix $(0.1,1.1 \mathrm{~ns})$ at room temperature. The same effect is observed for $\mathbf{2 b}$ in toluene $(0.069,0.55 \mathrm{~ns})$ versus PVA $(0.1,1.1 \mathrm{~ns})$ and intermediate results are observed in ethylene glycol, which has intermediate viscosity (Table 2 ). By contrast with the sterically unhindered $\mathbf{2 a}$ and $\mathbf{2 b}$, the respective mesityl and $o$-tolyl complexes $\mathbf{2 d}$ and $\mathbf{2 c}$, which have internal steric hindrance to aryl rotation, show virtually no change with medium viscosity, namely fluorescence yields of 0.89-0.93 and lifetimes of 5.8-6.9 ns in both toluene and PVA. Parallel but even more dramatic effects on the fluorescence properties of dipyrrins with and without internal steric hindrance to 5-aryl rotation are found with temperature, as is described in the following.

Temperature Dependence of the Fluorescence Yield. The effect of temperature on the fluorescence behavior of the phenyl-substituted complex 2a was examined in parallel with the mesityl-substituted analogue 2d. Solid PVA was used as the medium in both cases because (1) PVA is transparent across the temperature range studied ( 295 to $78 \mathrm{~K}$ ), (2) discontinuities at the freezing point of a solution sample are avoided, and (3) changes in viscosity with temperature are small compared to a solution sample, affording a more clear effect of temperature (thermal energy). Figure 3 shows the results. The integrated fluorescence of unhindered 2a in PVA increases about 6-fold when the temperature is lowered from 295 to 78 K. In contrast, the emission from hindered $\mathbf{2 d}$ appears to increase slightly with the same reduction in temperature. [There is little $(<20 \%)$ temperature dependence of the absorbance at the 450 or $455 \mathrm{~nm}$ excitation wavelength in either sample.]

An apparent activation energy on the order of $1000 \mathrm{~cm}^{-1}$ is obtained from an Arrhenius analysis. It must be born in mind that this activated phenomenon may not simply reflect the energy for photoexcited 2a to surmount a barrier for phenyl rotation. Instead, this is likely to represent a rather low-energy torsional motion $\left(<50 \mathrm{~cm}^{-1}\right)$, coupled with nonplanar distortion of the dipyrrin framework. These low-energy motions are no doubt populated to very high quantum levels, especially at ambient temperature. Hence the apparent activation energy may represent in part the quantum-level dependence of tunneling through a torsional barrier, and not an actual barrier crossing.

Regardless of the precise physical significance of the apparent activation energy, the key finding is that the fluorescence yield of the phenyl-substituted boron dipyrrin lacking internal steric constraint (2a) increases significantly when thermal energy is reduced. The value at 78 
$\mathrm{K}\left(\Phi_{\mathrm{f}} \sim 0.35\right)$ has climbed to 30-50\% of that of the mesityl-substituted analogue, which contains internal hindrance to aryl-ring rotation (2d). These observations are another indication that excited-state conformation excursions are a key process in driving the observed fluorescent behavior of aryl-substituted boron-dipyrrin dyes.

Transient Absorption Spectra and Kinetics. Figures 4 and 5 give representative transient absorption spectra and kinetic data for $\mathbf{2 a}, \mathbf{2} \mathbf{b}$, and $\mathbf{2 d}$ in toluene at room temperature, employing $\sim 100 \mathrm{fs}$ excitation flashes at $\sim 485 \mathrm{~nm}$. Additional data are given in the Supporting Information. Comparison with the static absorption (solid) and fluorescence (dashed) spectra for 2a in Figure 4B indicates that the transient absorption difference spectra in Figure 4A are comprised of bleaching of the $S_{0} \rightarrow S_{1}$ ground-state absorption band at $\sim 505 \mathrm{~nm}$ plus a tail to longer wavelengths representing $S_{1} \rightarrow S_{0}$ stimulated emission (i.e., gain in the white-light probe pulse in the fluorescence region). The spectra have decayed completely to the $\Delta \mathrm{A}=0$ baseline by $2 \mathrm{~ns}$, indicating complete deactivation to the ground state and insignificant formation of a longer-lived metastable state such as the triplet $\left(\mathrm{T}_{1}\right)$ excited state. Given the very small fluorescence yield $(\sim 0.06$, Table 2$)$, these combined data indicate that the decay of photoexcited $2 \mathrm{a}$ in toluene occurs predominantly by $\mathrm{S}_{1} \rightarrow \mathrm{S}_{0}$ nonradiative internal conversion.

The kinetic traces in Figure 4C show a major ( $>70 \%$ ) component with a time constant of $\sim 400$ ps to the decay of both ground state bleaching $(505 \mathrm{~nm})$ and stimulated emission $(545 \mathrm{~nm})$; this value agrees well with the $S_{1}$ lifetime of $0.45 \mathrm{~ns}$ observed via fluorescence decay (Table 2). The decay of ground-state-absorption bleaching also shows a component with a time constant of $\sim 1$ ps along with a contribution from a third phase having a time constant of $\sim 10$ ps. The $\sim 10$ ps phase also contributes in the stimulated-emission region. Thus, these two fast phases represent a combination of rapid deactivation to the ground state (in addition to the dominant 380 ps phase) and conformational evolution on the $S_{1}$ surface with consequent spectral changes. These results corroborate the biphasic kinetics observed previously for $\mathbf{2 a}$ and $\mathbf{1}$ in toluene, ${ }^{11}$ and reveal at least one additional contribution to the excited-state dynamics.

Similar results to those found for the phenyl-substituted complex $\mathbf{2 a}$ are also observed for the 4-tert-butylphenyl analogue $\mathbf{2 b}$ (Figure 5A). In particular, deactivation to the ground state is complete by $2 \mathrm{~ns}$, and occurs predominantly with a time constant of $500 \mathrm{ps}$ that is in good agreement with the value ( $0.55 \mathrm{~ns}$ ) obtained by fluorescence decay (Table 2$)$. In contrast, a much longer deactivation time is observed for the mesityl-substituted 2d (Figure 5B). For this complex, the ground-state recovery is only about one-half complete by $3.5 \mathrm{~ns}$. When the decay profile observed over the $\sim 4 \mathrm{~ns}$ limit of this spectrometer is fit to an exponential decay function containing $\Delta \mathrm{A}=0$ at long-time asymptote (either fixed or in a free fit), a time constant of $\sim 6$ $\mathrm{ns}$ is obtained. This value is in good agreement with the $\mathrm{S}_{1}$ lifetime of $6.6 \mathrm{~ns}$ observed by fluorescence decay. These combined results indicate that the $S_{1}$ decay for $\mathbf{2 d}$ leads predominantly to repopulation of the ground state with little triplet formation. This result is expected since the fluorescence yield of this compound of 0.93 , which returns the excited molecules to the ground state via this emissive route.

Thus, the transient absorption data are fully consistent with the results of the static spectroscopy and fluorescence lifetime measurements and indicate that the $S_{1}$ excited state of sterically hindered boron dipyrrins $2 \mathbf{d}$ (and $\mathbf{2 c}$ ) decay predominantly ( $\geq 90 \%)$ by fluorescence emission whereas the unhindered analogues $\mathbf{2 a}$ (and $\mathbf{2 b}$ ) deactivate predominantly $(\geq 90 \%)$ by nonradiative internal conversion to the ground state.

Theoretical Analysis. Nature of the Lowest Excited Singlet States and Assignment of the Optical Transitions. The energies of the excited states of the boron-dipyrrin dyes were calculated using the symmetry-adapted cluster configuration interaction (SACCI) method. A comparison of the SACCI excited singlet state manifolds with the absorption spectra of $\mathbf{4}$ and 
2a is shown in Figure 6. In general, SACCI with single and double configuration interaction reproduces the absorption spectra of these compounds. The results support the notion that the lowest-lying excited singlet state is a strongly allowed $B_{2}$ or $B_{2}$-like state. As shown in the state correlation diagram (Figure 7), the ordering of the lowest three excited singlet states $\left(B_{2}, 1 A_{1}, 2 B_{2}\right)$ is not sensitive to substitution and aryl rotation. However, the position of the $2 A_{l}$ excited state is very sensitive to rotation of the aryl ring. When this ring is phenyl and is rotated to the minimum energy position $\left(\Phi=50^{\circ}\right)$, the $2 A_{l}$ state drops dramatically $(\sim 10 \mathrm{kK})$ in energy as shown in the insert in the bottom panel of Figure 6.

We conclude that the strong absorption band at $30 \mathrm{kK}(\sim 330 \mathrm{~nm})$ is due mainly to the $2 A_{1}$ transition and that the less intense $2 B_{2}$ transition is associated with the smaller band observed at $26 \mathrm{kK}(\sim 380 \mathrm{~nm})$. Spectral fitting indicates that the lower of these two bands has an oscillator strength roughly half that of the higher energy feature, in reasonable agreement with the calculated SACCI-CISD oscillator strengths for the $2 A_{1}\left(f_{\text {calc }}=0.37\right)$ and $2 B_{2}\left(f_{\text {calc }}=\right.$ 0.18 ) states. [For reference, the strongly allowed lowest energy $B_{2}$ state has a calculated oscillator strength of 0.44.] This assessment of the contribution of the $2 A_{l}$ state, along with $1 A_{l}$ and $2 B_{2}$ states, to the absorption in the $300-400 \mathrm{~nm}$ region is consistent with several observations. First, the absorption contour in this region for a given solvent is dependent on the nature (or presence) of the 5-aryl ring (Figures 2A-E). Since different dihedral angles are expected for the different types of complexes (e.g., hindered versus unhindered) on the basis of the ground-state structural studies (Table 1 and Figure 1), the contribution of the $2 A_{l}$ to absorption in the 300-400 $\mathrm{nm}$ region will differ. Second, the calculations indicate that the $2 A_{1}$ state has a somewhat larger dipole moment than the ground state (the $B_{2}$ states have a slightly smaller dipole moment than the ground state). Thus, an increase in solvent polarity will tend to cause the aryl ring to rotate to enhance the excited-state dipole moment and thereby increase solvent stabilization. According to the calculations, this will also increase the oscillator strength and contribution of the $2 A_{I}$ state to the near-UV absorption contour. These predictions are consistent with the solvent polarity effects on the absorption spectra (Figure 2E and Supporting Information). Third, calculations indicate that the $A_{l}$ transitions are polarized perpendicular to the $B_{2}$ transitions (see Table 2 described below). Thus, a contribution of the $2 A_{2}$ (and weaker $1 A_{2}$ ) transition to the absorption contour in the 300-400 nm region (and not simply just the $2 B_{2}$ transition) is consistent with the finding that the polarization in this region tends toward perpendicularity to the $\mathrm{S}_{0} \leftrightarrow \mathrm{S}_{1}\left(1 B_{2}\right)$ absorption and fluorescence bands.

In summary, the calculations and experimental results are consistent with a lowest lying $\mathrm{B}_{2}$ state for all the compounds investigated, and the fact that the lowest three excited singlet states are $A_{1}$ or $B_{2}$ symmetry. Interestingly, the separation between the lowest and the second excited singlet states is relatively large, regardless of substitution or aryl geometry.

Configurational Characteristics of the Lowest Excited Singlet States. The configurational properties of the lowest-lying $B_{2}$ excited singlet state in the phenyl-substituted dye 2a are presented in Figure 8, on the basis of SACCI calculations using full single and double CI. Note that while Figures 6 and ${ }^{7}$ display calculated excited-state transition energies using vertical bars (Figure 6) or horizontal lines (Figure 7), the horizontal lines in Figure 8 show the HartreeFock energies of the molecular orbitals. The symmetry of the closed-shell ground state of a molecule with $\mathrm{C}_{2 \mathrm{v}}$ symmetry is $A_{1}$, and the $\pi$ molecular orbitals of the boron-dipyrrin framework have $a_{2}$ or $b_{1}$ symmetry. For all the molecules investigated here, the lowest unoccupied molecular orbital (LUMO) has $b_{1}$ symmetry and is notably isolated in energy from the other unoccupied orbitals (Figure 8). Thus, the dominant configuration for most of the lowlying excited states involves excitation from one of the filled orbitals into the $b_{1}$ LUMO. The situation is illustrated in Table 3 , which provides a symmetry analysis of the transition polarization ( $\mathrm{x}$ and $\mathrm{y}$ are in plane) and the lowest energy configurations on the basis of the boron-dipyrrin orbitals. (The $A_{2}$ state is rigorously forbidden.) Since the highest occupied 
molecular orbital (HOMO) has a 2 symmetry, inspection of Table 3 , along with the orbital energies in Figure 8 provides a clear picture of why the lowest singlet state is an allowed $B_{2}$ state and why this state is well separated in energy from the second excited singlet state.

We now explore the nature of the lowest $B_{2}$ excited singlet state in 2a in more detail. The calculation at the lower left of Figure 8 shows the composition of the transition with the 5phenyl group rotated orthogonal to the plane of the boron-dipyrrin framework, while the calculation at lower right shows the transition with the phenyl group rotated into the dipyrrin plane. The symmetry labels for the latter case are approximate. Single arrows represent single excitations and double arrows represent double excitations, with the percentage contribution for each excitation shown within the yellow circles. The sum of the percentages is shown to the right of the set, and in both cases, more than $90 \%$ of the state configurational character is represented by the seven configurations. More interestingly, the rotation of the phenyl group has only minor impact on the configurational distribution, and the lowest $B_{2}$ singlet state is described well as a simple HOMO to LUMO transition. As shown in the top two rows of panels in Figure 8, the HOMO is localized in the boron-dipyrrin framework irrespective of phenylgroup geometry. In contrast, the LUMO is localized in the boron-dipyrrin framework when the phenyl group is orthogonal, but this orbital is extensively delocalized into the phenyl ring upon rotation of the latter into the plane of the dipyrrin system. This delocalization of electron density is responsible for the decrease in the LUMO energy and the corresponding decrease in the transition energy upon aryl ring rotation (Figure 7). As is shown in Figure 8, the LUMO is the single most important orbital in defining the configurational characteristics of the lowestlying, strongly allowed $\mathrm{B}_{2}$ state because the LUMO participates in over $90 \%$ of the configurational description.

The same strongly allowed $B_{2}$ character of the lowest excited state is found when the fluorine atoms of 2a are replaced by ethyl groups, which were substituted for $n$-butyl groups of $\mathbf{2 a -}$ $\mathbf{B u}_{2}$ to make the calculation more efficient. As is shown in Figure 7 and described in the Supporting Information, the calculations also indicate that the alkyl groups should preferentially stabilize the $A_{1}$ states, thereby reversing the ordering of the second and third excited states $\left(1 \mathrm{~A}_{1}\right.$ with $\left.2 \mathrm{~B}_{1}\right)$, with consequences on the absorption contour between 300 and $400 \mathrm{~nm}$ (see Figure 2). The calculations showed no differences in the potential energy surfaces for the ground or lowest excited state compared to $\mathbf{2 a}$.

Franck-Condon Activity in the Optical Spectra. The strongly allowed low-energy absorption bands in these compounds are unusually sharp, exhibiting full-widths at half-maxima of less than $1200 \mathrm{~cm}^{-1}$ (Figures 2 and 6). This sharpness is observed regardless of the substituents at the 5-position or on the pyrrole rings. Comparisons of the calculated ground- and excited-state geometries provide a perspective on this observation. Simply stated, the ground-and lowestexcited $\mathrm{B}_{2}$ states have virtually identical geometries in the Franck-Condon region. To a first approximation, only $A_{l}$ or $A_{l}$-like vibrational modes are Franck-Condon active and these modes are confined largely to the ring system. The promoting modes in absorption and fluorescence of $\mathbf{4}$ involve in-plane breathing vibrations of the ring atoms coupled to a N-B-N angular mode (see illustrations in Supporting Information). These modes are relatively insensitive to whether there is hydrogen or an aryl ring at the 5-position. The possible exceptions to this rule involve the phenyl and 4-tert-butylphenyl complexes $\mathbf{2 a}$ and $\mathbf{2 b}$, respectively, which have no internal hindrance to aryl rotation. As described below, 2a (and by comparison $\mathbf{2 b}$ ) has a lowest excited singlet state geometry that is significantly different than the ground-state geometry (Figures 9A and B). Nevertheless, this significant change in geometry generates only a minor increase in the optical bandwidths (Figure 6). There are two reasons for this observation. First, the LUMO does not expand into the aryl group significantly until the ring approaches within $30^{\circ}$ of the planar geometry, an orientation that is never explored in the ground state at ambient temperature because the LUMO is populated only in the excited 
state. Thus, the relaxed excited-state geometry is outside of the Franck-Condon active region. Second, the vibration that is responsible for dihedral relaxation of the phenyl group is a lowfrequency torsional mode that has poor vibrational overlap with the ground-state modes regardless of geometry. The result is a Franck-Condon distribution that is dominated by inplane $\mathrm{A}_{1}$-like modes of the dipyrrin framework in all of the compounds investigated here. This observation provides insight into the fluorescence quantum yields in those compounds (2d, 2c) wherein aryl rotation is restricted (Table 2).

Ground and Excited State Potential Energy Surfaces. Rotation of the phenyl or aryl ring relative to the boron-dipyrrin plane is the key conformational degree of freedom that determines the photophysical properties of these molecules. However, as the aryl group rotates, interactions between the $o$-aryl groups and the hydrogen atoms of the dipyrrin framework forces the latter to pucker, as shown in Figures 9A and B. In order to describe the rotation of the aryl group with internal consistency, we use two bond centroids on the boron-dipyrrin ring ( $\mu$ and $\nu>$, Figure 9A) and two bond centroids on the aryl group ( $\lambda$ and $\sigma)$ to define the dihedral angle. The potential-energy surfaces for rotation of the phenyl (2a, Figure 9C), mesityl (2d, Figure 10) and $o$-tolyl (2c, Supporting Information) groups are plotted with reference to this torsional angle. Furthermore, only the lowest energy conformation as a function of this angle is plotted. Computationally, there is a tendency of the ring to pucker in an asymmetric fashion as the aryl group approaches coplanarity with the dipyrrin framework. The asymmetry depends upon the initial conformation and is an artificial component of the minimization process. In reality, an ambient temperature system will explore many different conformations during the aryl rotation so our decision to plot only the lowest-energy conformation is appropriate.

Excited-state Dynamics for $\mathbf{2 a}$. The most interesting potential energy surfaces are calculated for 2a and are shown in Figure 9C. This molecule is calculated to have a barrier to phenyl rotation in the ground state of approximately $50 \mathrm{~kJ} / \mathrm{mol}$; the lowest energy conformation is one in which the phenyl group is twisted to have an angle of about $55^{\circ}\left(145^{\circ}\right)$, namely $35^{\circ}$ from perpendicularity. This result is in good agreement with the average dihedral angle of $\sim 60^{\circ}$ determined from the X-ray structure (Table 1). The phenyl group in the lowest excited triplet state has a barrier to rotation of $25 \mathrm{~kJ} / \mathrm{mol}$, roughly half that observed in the ground state, and displays a local minimum in the planar geometry (Figure 9). In contrast, the lowest excited singlet state displays a potential surface dramatically different, with a minimum geometry at a dihedral angle of $180^{\circ}$ (Figures $9 \mathrm{~A}$ and $\mathrm{B}$ ). The origin of this potential-surface minimum is intimately associated with the delocalization of the LUMO as discussed above and as shown in Figure 8. We have discussed the basic driving forces behind this phenomenon in a previous paper. ${ }^{11}$ However, our previous theoretical treatment used semi-empirical methods with limited double CI to explore the excited-state surface. The higher level theory used in this study provides a higher correlated singlet excited-state surface, and reveals that the $S_{1}$ surface provides virtually no barrier to rotation of the phenyl group towards planarity in $\mathbf{2 a}$ (compare Figure 9 with Figures 7 and 8 of Ref 11). The new calculations provide a clear perspective on why $\mathbf{2 a}$ (and $\mathbf{2 b}$ ) has a negligible fluorescence quantum yield. The barrier-less or nearly barrierless $\mathrm{S}_{1}$ surface provides a rapid transfer of the excited-state population to the delocalized puckered conformation. In this form, the phenyl group has rotated to a dihedral angle of $180^{\circ}$, where there is a new minimum in the excited-state surface accompanied by enhanced electron delocalization. The $S_{1}$ excited state in this conformation has efficient coupling to the ground state via nonradiative processes. If any emission were to occur, it would be in the infrared.

We note with interest that the triplet excited state does not share a minimum energy delocalized puckered conformation. The factors underlying this observation are described in the Supporting Information. The time-resolved absorption data described above indicate that internal conversion of the lowest excited singlet state is so facile as to circumvent any appreciable intersystem crossing to produce the triplet excited state. 
Excited-state Dynamics for $\mathbf{2 d}$. The ground and first excited singlet state surfaces for $\mathbf{2 d}$ are shown in Figure 10. The $o$ - and $o^{\prime}$-methyl groups cause increased repulsion between the mesityl group and the boron-dipyrrin framework to such an extent that the lowest-energy ground state conformation is one in which the mesityl ring lies essentially orthogonal to the dipyrrin framework. As one might anticipate, the delocalized puckered state in which the mesityl ring rotates approximately coplanar with the dipyrrin is not the lowest energy excited singlet state conformation (as it was for 2a), but lies $60 \mathrm{~kJ} / \mathrm{mol}$ higher in energy than the orthogonal or nearly orthogonal conformations. We conclude that excitation into the excited singlet manifold of $\mathbf{2 d}$ generates only a modest change in the mesityl group orientation, and that fluorescence from the relaxed $S_{1}$ state should be efficient. This prediction is in excellent agreement with the findings from static and time-resolved optical spectroscopy.

Excited-state Dynamics for $\mathbf{2 c}$. The ground and first excited singlet state surfaces for $\mathbf{2 c}$ are shown in the Supporting Information. The $\mathbf{2 c}$ surface is qualitatively identical to that observed for $\mathbf{2 d}$ but has some interesting features in terms of local minima that are unlikely to have an observable impact on the excited-state dynamics. These conclusions are in keeping with the finding that the photophysical behavior of $o$-tolyl-substituted $\mathbf{2 c}$ is virtually identical to that of 2d. Thus, the presence of only one $o$-methyl group on the aryl ring provides sufficient hindrance to internal rotation to afford the substantial change in excited-state dynamics and emission properties compared to the unhindered analogues.

\section{Conclusions}

A family of 5-aryl-substituted boron-dipyrrin dyes has been synthesized and then characterized by X-ray diffraction, photophysical studies, and theory. The results demonstrate the dominant role of aryl rotation in governing the excited-state dynamics and fluorescence properties of these dyes. The results should facilitate the further use of this class of dyes and related systems as optical probes in the life sciences and other applications.

\section{Experimental Section}

\section{Synthesis}

General. ${ }^{1} \mathrm{H}$ NMR spectra $(400 \mathrm{MHz})$ and ${ }^{13} \mathrm{C}$ NMR spectra $(100 \mathrm{MHz})$ were collected in $\mathrm{CDCl}_{3}$ unless noted otherwise. Absorption spectra and fluorescence spectra (and yields) were collected in toluene at room temperature unless noted otherwise. Adsorption column chromatography was performed using flash silica gel. Silica gel ( $40 \mu \mathrm{m}$ average particle size) was used for column chromatography. Anhydrous $\mathrm{CH}_{2} \mathrm{Cl}_{2}$ was purchased from Aldrich. All other chemicals were reagent grade and were used as received. Compounds 4 (BDPY 3921) and 5 (BDPY 3922) were purchased from Molecular Probes Inc.

Noncommercial compounds. Compounds 5-(o-tolyl)dipyrromethane (7c) ${ }^{4} 5$-phenyldipyrrin (8a),,${ }^{6,12}$ 5-(4-tert-butylphenyl)dipyrrin (8b), ${ }^{12}$ and 5-mesityldipyrrin (8d) ${ }^{12}$ were prepared according to literature procedures.

$N, N^{\prime}$-difluoroboryl-5-phenyldipyrrin (2a). A solution of $\mathbf{8 a}(440 \mathrm{mg}, 2.00 \mathrm{mmol})$ in $\mathrm{CH}_{2} \mathrm{Cl}_{2}(20 \mathrm{~mL})$ was treated with TEA $\left(2.79 \mathrm{~mL}, 20.0 \mathrm{mmol}, 10\right.$ equiv) and $\mathrm{BF}_{3} \cdot \mathrm{O}(\mathrm{Et})_{2}(2.53$ $\mathrm{mL}, 20.0 \mathrm{mmol}, 10$ equiv) at room temperature. After $30 \mathrm{~min}$, TLC analysis (silica, $\mathrm{CH}_{2} \mathrm{Cl}_{2}$ ) indicated complete consumption of the starting material. The reaction mixture was washed with saturated aqueous $\mathrm{NaHCO}_{3}(100 \mathrm{~mL} \times 3)$. The organic layer was dried $\left(\mathrm{Na}_{2} \mathrm{SO}_{4}\right)$, concentrated, and chromatographed [silica, $\mathrm{CH}_{2} \mathrm{Cl}_{2}$ /hexanes (1:1)], affording a brown oil. Addition of $\mathrm{CH}_{2} \mathrm{Cl}_{2} /$ hexanes precipitated a red-orange solid (105 mg, 19\%): $\mathrm{mp} 99-100^{\circ}$ $\mathrm{C} ;{ }^{1} \mathrm{H}$ NMR $\Delta 6.55(\mathrm{~d}, J=3.6 \mathrm{~Hz}, 2 \mathrm{H}), 6.94(\mathrm{~d}, J=3.6 \mathrm{~Hz}, 2 \mathrm{H}), 7.51-7.60(\mathrm{~m}, 5 \mathrm{H}), 7.93-7.97$ (m, 2H); ${ }^{13}$ C NMR $\Delta 128.4,130.44,130.74,131.6,133.7,134.9,144.0,147.3$; FABMS obsd 
268.0974, calcd 268.0983 $\left(\mathrm{C}_{15} \mathrm{H}_{11} \mathrm{BF}_{2} \mathrm{~N}_{2}\right)$; Anal. Calcd: $\mathrm{C}, 67.21 ; \mathrm{H}, 4.14 ; \mathrm{N}, 10.45$. Found: $\mathrm{C}, 67.28 ; \mathrm{H}, 4.29 ; \mathrm{N}, 10.32 ; \lambda_{\mathrm{abs}} 504 \mathrm{~nm}, \lambda_{\mathrm{em}} 521 \mathrm{~nm}, \Phi_{\mathrm{f}}=0.062$.

$N, N^{\prime}$-difluoroboryl-5-(4-tert-butylphenyl)dipyrrin (2b). Following the procedure for 2a, the reaction of $\mathbf{8 b}(1.10 \mathrm{~g}, 4.00 \mathrm{mmol})$ in $\mathrm{CH}_{2} \mathrm{Cl}_{2}(40 \mathrm{~mL})$ with TEA $(5.58 \mathrm{~mL}, 40.0 \mathrm{mmol}, 10$ equiv) and $\mathrm{BF}_{3} \cdot \mathrm{O}(\mathrm{Et})_{2}(5.07 \mathrm{~mL}, 40.0 \mathrm{mmol}, 10$ equiv) followed by workup and chromatography [silica, $\mathrm{CH}_{2} \mathrm{Cl}_{2}$ /hexanes (1:1)] afforded an orange solid. The solid was suspended in methanol and sonicated. The suspension was filtered, affording a red-orange solid (0.625 g, 50\%): $\mathrm{mp} 169-170{ }^{\circ} \mathrm{C} ;{ }^{1} \mathrm{H}$ NMR $\Delta 1.40(\mathrm{~s}, 9 \mathrm{H}), 6.52-6.57(\mathrm{~m}, 2 \mathrm{H}), 7.00(\mathrm{~d}, J=3.6$ $\mathrm{Hz}, 2 \mathrm{H}), 7.52(\mathrm{~d}, J=8.4 \mathrm{~Hz}, 2 \mathrm{H}), 7.54(\mathrm{~d}, J=8.4 \mathrm{~Hz}, 2 \mathrm{H}), 7.91-7.95(\mathrm{~m}, 2 \mathrm{H}) ;{ }^{13} \mathrm{C}$ NMR $\Delta$ 31.1, 34.9, 118.2, 125.4, 130.44, 130.85, 131.6, 134.8, 143.5, 147.6, 154.4; FABMS obsd 324.1620, calcd $324.1609\left(\mathrm{C}_{19} \mathrm{H}_{19} \mathrm{BF}_{2} \mathrm{~N}_{2}\right)$; Anal. Calcd: C, 70.39; H, 5.91; N, 8.64. Found: $\mathrm{C}$, $70.39 ; \mathrm{H}, 5.87 ; \mathrm{N}, 8.59 ; \lambda_{\text {abs }} 502 \mathrm{~nm}, \lambda_{\text {em }} 522 \mathrm{~nm}, \Phi_{\mathrm{f}}=0.069$.

$\boldsymbol{N}, N^{\prime}$-difluoroboryl-5-o-tolyldipyrrin (2c). Following the procedure for $\mathbf{2 a}$, the reaction of 8c $(0.214 \mathrm{~g}, 0.912 \mathrm{mmol})$ in $\mathrm{CH}_{2} \mathrm{Cl}_{2}(10 \mathrm{~mL})$ with TEA $(1.27 \mathrm{~mL}, 0.914 \mathrm{mmol}, 10$ equiv) and $\mathrm{BF}_{3} \cdot \mathrm{O}(\mathrm{Et})_{2}(1.16 \mathrm{~mL}, 0.915 \mathrm{mmol}, 10$ equiv) followed by workup, chromatography [silica, $\mathrm{CH}_{2} \mathrm{Cl}_{2} /$ hexanes (1:1)], and recrystallization (methanol/water) afforded an orange solid (0.175 g, 68\%): $\mathrm{mp} 135{ }^{\circ} \mathrm{C} ;{ }^{1} \mathrm{H}$ NMR $\Delta 2.25(\mathrm{~s}, 3 \mathrm{H}), 6.50(\mathrm{~d}, J=3.6 \mathrm{~Hz}, 2 \mathrm{H}), 6.72(\mathrm{~d}, J=3.6 \mathrm{~Hz}$, $2 \mathrm{H}), 7.27-7.31(\mathrm{~m}, 2 \mathrm{H}), 7.34(\mathrm{~d}, J=8.0 \mathrm{~Hz}, 1 \mathrm{H}), 7.41-7.45(\mathrm{~m}, 1 \mathrm{H}), 7.92-7.95(\mathrm{~m}, 2 \mathrm{H})$; ${ }^{13}$ C NMR $\Delta 20.1,118.6,125.3,129.7,129.8,130.4,131.0,136.3$, 144.4; FABMS obsd 282.1146, calcd $282.1140\left(\mathrm{C}_{16} \mathrm{H}_{13} \mathrm{BF}_{2} \mathrm{~N}_{2}\right)$; Anal. Calcd: C, 68.12; H, 4.64; N, 9.93. Found: $\mathrm{C}$, $67.95 ; \mathrm{H}, 4.79 ; \mathrm{N}, 9.73 ; \lambda_{\mathrm{abs}} 503 \mathrm{~nm}, \lambda_{\mathrm{em}} 518 \mathrm{~nm}, \Phi_{\mathrm{f}}=0.93$.

$N, N^{\prime}$-difluoroboryl-5-mesityldipyrrin (2d). Following the procedure for $2 \mathrm{a}$, the reaction of 8d (1.05 g, $4.00 \mathrm{mmol})$ in $\mathrm{CH}_{2} \mathrm{Cl}_{2}(40 \mathrm{~mL})$ with TEA (5.58 mL, $40.0 \mathrm{mmol}, 10$ equiv) and $\mathrm{BF}_{3} \cdot \mathrm{O}(\mathrm{Et})_{2}(5.07 \mathrm{~mL}, 40.0 \mathrm{mmol}, 10$ equiv) followed by workup and chromatography [silica, $\mathrm{CH}_{2} \mathrm{Cl}_{2}$ hexanes (1:1)] afforded an orange solid. The solid was suspended in methanol and sonicated. The suspension was filtered, affording a red-orange solid $(0.677 \mathrm{~g}, 52 \%)$ : $\mathrm{mp} 168-$ $169^{\circ} \mathrm{C} ;{ }^{1} \mathrm{H}$ NMR $\Delta 2.10(\mathrm{~s}, 6 \mathrm{H}), 2.37(\mathrm{~s}, 3 \mathrm{H}), 6.47(\mathrm{~d}, J=4.0 \mathrm{~Hz}, 2 \mathrm{H}), 6.68(\mathrm{~d}, J=4.0 \mathrm{~Hz}$, 2H), 6.96 (s, 2H), 7.90-7.94 (m, 2H); ${ }^{13} \mathrm{C}$ NMR $\Delta$ 19.9, 21.1, 118.5, 128.1, 129.6, 130.1, 135.3, 136.2, 138.8, 144.2, 147.6; FABMS obsd 310.1447, calcd $310.1453\left(\mathrm{C}_{18} \mathrm{H}_{17} \mathrm{BF}_{2} \mathrm{~N}_{2}\right)$; Anal. Calcd: C, 69.71; H, 5.52; N, 9.03. Found: C, 69.50; H, 5.60; N, 8.90; $\lambda_{\text {abs }} 503$ nm, $\lambda_{\text {em }} 517$ $\mathrm{nm}, \Phi_{\mathrm{f}}=0.93$.

$\boldsymbol{N}, \boldsymbol{N}^{\prime}$-(Dimethylboryl)-5-phenyldipyrrin (2a-Me $)$. A 20-mL reaction vial was charged with 8a (72 mg, $0.32 \mathrm{mmol})$, anhydrous $\mathrm{CH}_{2} \mathrm{Cl}_{2}(3.6 \mathrm{~mL})$, TEA $(89 \mu \mathrm{L}, 0.64 \mathrm{mmol})$ and a magnetic stirring bar. The vessel was capped with a septum and bromodimethylborane $(63 \mu \mathrm{L}, 0.64$ $\mathrm{mmol}$ ) was added via syringe. The reaction mixture was stirred for $90 \mathrm{~min}$. The reaction mixture was then concentrated to dryness. The residue was dissolved in a minimum of $\mathrm{CHCl}_{3}$ and chromatographed (silica, $\mathrm{CHCl}_{3}$ ). The product eluted as an orange band. Fractions containing the pure product were concentrated, providing an orange solid (77 mg, 89\%): $\mathrm{mp} \mathrm{124-125^{ \circ }}$ $\mathrm{C} ;{ }^{1} \mathrm{H}$ NMR $\Delta 0.20(\mathrm{~s}, 6 \mathrm{H}), 6.49-6.53(\mathrm{~m}, 2 \mathrm{H}), 6.83-6.87(\mathrm{~m}, 2 \mathrm{H}), 7.47-7.63(\mathrm{~m}, 5 \mathrm{H}), 7.67-$ $7.71(\mathrm{~m}, 2 \mathrm{H}) ;{ }^{13} \mathrm{C}$ NMR $\Delta 14.5$ (brs), 117.5, 127.96, 128.3, 130.1, 130.6, 133.4, 135.2, 142.8, 146.5; LDMS obsd 259.6, calcd $260.2\left(\mathrm{C}_{17} \mathrm{H}_{17} \mathrm{BN}_{2}\right) ; \lambda_{\text {abs }} 495 \mathrm{~nm}, \lambda_{\mathrm{em}} 518 \mathrm{~nm}, \Phi_{\mathrm{f}}=0.008$.

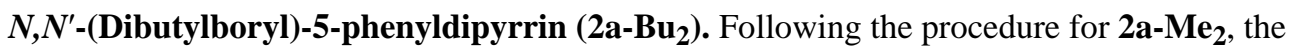
reaction of $8 \mathbf{a}(75 \mathrm{mg}, 0.34 \mathrm{mmol})$ in $\mathrm{CH}_{2} \mathrm{Cl}_{2}(1.0 \mathrm{~mL})$ with TEA $(95 \mu \mathrm{L}, 0.68 \mathrm{mmol})$ and dibutylboron triflate $\left(680 \mu \mathrm{L}\right.$ of a $1.0 \mathrm{M}$ solution in $\mathrm{CH}_{2} \mathrm{Cl}_{2}, 0.68 \mathrm{mmol}$ ) was carried out for 4 $\mathrm{h}$, whereupon TLC analysis (silica, hexanes) indicated that the reaction was complete. The mixture was diluted with $4 \mathrm{~mL}$ of hexanes and chromatographed (silica, hexanes w/2\% ethyl acetate). The product eluted as a yellow band. Fractions containing the pure product were concentrated, providing a sticky orange resin $(30.0 \mathrm{mg}, 26 \%) .{ }^{1} \mathrm{H}$ NMR analysis indicated the 
presence of a small impurity ( $\sim 5 \%)$. Additional silica gel chromatography, even in neat hexanes or pentane could not remove this species. ${ }^{1} \mathrm{H}$ NMR $\Delta 0.58-0.85(\mathrm{~m}, 10 \mathrm{H}), 0.91-1.15(\mathrm{~m}, 2 \mathrm{H})$, $1.50-1.80(\mathrm{~m}, 3 \mathrm{H}), 1.53-1.70(\mathrm{~m}, 1 \mathrm{H}), 2.80-2.98(\mathrm{~m}, 2 \mathrm{H}), 6.45(\mathrm{~d}, J=4.4 \mathrm{~Hz}, 0.5 \mathrm{H}), 6.46-$ $6.49(\mathrm{~m}, 0.5 \mathrm{H}), 6.52-6.58(\mathrm{~m}, 1 \mathrm{H}), 6.65-6.67(\mathrm{~m}, 0.5 \mathrm{H}), 6.82-6.86(\mathrm{~m}, 1.5 \mathrm{H}), 7.43-7.57(\mathrm{~m}$, $5 \mathrm{H}), 7.58-7.63(\mathrm{~m}, 2 \mathrm{H})$; LDMS obsd 343.0, calcd $344.2\left(\mathrm{C}_{23} \mathrm{H}_{29} \mathrm{BN}_{2}\right) ; \lambda_{\text {abs }} 498 \mathrm{~nm}, \lambda_{\mathrm{em}} 521$ $\mathrm{nm}, \Phi_{\mathrm{f}}=0.008$. The relative complexity of the ${ }^{1} \mathrm{H}$ NMR signature obtained is not reflective of the $\mathrm{C}_{2 \mathrm{v}}$ symmetry expected for this compound; however, such complexity is not a consequence of the tiny amount of impurity.

$N, N^{\prime}$-(Dimethylboryl)-5-mesityldipyrrin (2d-Me $)_{2}$. Following the procedure for $\mathbf{2 a}-\mathbf{M e}_{2}$, the reaction of $8 \mathbf{d}(50.0 \mathrm{mg}, 0.191 \mathrm{mmol})$ in $\mathrm{CH}_{2} \mathrm{Cl}_{2}(2.0 \mathrm{~mL})$ with TEA $(265 \mu \mathrm{L}, 1.91 \mathrm{mmol})$ and bromodimethylborane ( $186 \mu \mathrm{L}, 1.91 \mathrm{mmol})$ was carried out for $30 \mathrm{~min}$. Workup and chromatography [silica, hexanes/ $\mathrm{CH}_{2} \mathrm{Cl}_{2}(5: 1)$ ] afforded a sticky oil that solidified to an orange solid (50 mg, 87\%): $\mathrm{mp} 113{ }^{\circ} \mathrm{C} ;{ }^{1} \mathrm{H}$ NMR $\Delta 0.19$ (s, 6H), 2.11 (s, 6H), 2.38 (s, 3H), 6.43 (dd, $J=4.0 \mathrm{~Hz}, J=2.0 \mathrm{~Hz}, 2 \mathrm{H}), 6.60(\mathrm{dd}, J=4.4 \mathrm{~Hz}, J=0.8 \mathrm{~Hz}, 2 \mathrm{H}), 6.96(\mathrm{~s}, 2 \mathrm{H}), 7.63-7.68(\mathrm{~m}$, $2 \mathrm{H}),{ }^{13} \mathrm{C}$ NMR $\Delta 13.7$ (brs), 19.8, 21.1, 117.1, 126.3, 127.9, 131.1, 133.5, 136.3, 138.1, 142.2, 146.2 ;

FABMS obsd 303.2037, calcd $303.2033\left[(\mathrm{M}+\mathrm{H})^{+} ; \mathrm{M}=\mathrm{C}_{20} \mathrm{H}_{23} \mathrm{BN}_{2}\right] ; \lambda_{\text {abs }} 497 \mathrm{~nm}, \lambda_{\mathrm{em}} 514$ $\mathrm{nm}, \Phi_{\mathrm{f}}=0.40$.

$N, N^{\prime}$-(Dibutylboryl)-5-mesityldipyrrin $\left(\mathbf{2 d}-\mathbf{B u}_{2}\right)$. Following the procedure for $\mathbf{2 a}-\mathbf{M e}_{2}$, the reaction of $8 \mathbf{d}(60 \mathrm{mg}, 0.23 \mathrm{mmol})$ in $\mathrm{CH}_{2} \mathrm{Cl}_{2}(3.0 \mathrm{~mL})$ with TEA $(32 \mu \mathrm{L}, 0.23 \mathrm{mmol})$ and dibutylboron triflate ( $460 \mu \mathrm{L}$ of a $1.0 \mathrm{M}$ solution in $\mathrm{CH}_{2} \mathrm{Cl}_{2}, 0.46 \mathrm{mmol}$ ) was carried out for 1 $\mathrm{h}$, whereupon TLC analysis (silica, hexanes) indicated that the reaction was complete. The mixture was diluted with $3 \mathrm{~mL}$ of hexanes and chromatographed over a short silica column in hexanes. The product eluted as a yellow band. Fractions containing the product were concentrated and chromatographed over a second column in order to remove a pink impurity that followed closely behind the desired product. Fractions containing the pure product were concentrated, providing a sticky orange resin $(17 \mathrm{mg}, 19 \%):{ }^{1} \mathrm{H}$ NMR $\Delta 0.67-0.81(\mathrm{~m}, 14 \mathrm{H})$, 1.06-1.13 (m, 4H), 2.07 (s, 6H), 2.37 (s, 3H), 6.46-6.47 (m, 2H), 6.57-6.58 (m, 2H), $6.95(\mathrm{~s}$, 2H), 7.55-7.60 (m, 2H); ${ }^{13} \mathrm{C}$ NMR $\Delta 14.6,20.1,21.5,26.4,28.1,30.1,117.3,125.8,128.1$, 131.3, 134.5, 136.4, 138.2, 142.0, 146.5; LDMS obsd 386.2, calcd $386.3\left(\mathrm{C}_{26} \mathrm{H}_{35} \mathrm{BN}_{2}\right)$; Anal. Calcd for $\mathrm{C}_{26} \mathrm{H}_{35} \mathrm{BN}_{2}$ : C, 80.82; H, 9.13; N, 7.25; Found: C, 80.24; H, 9.41; N, 6.88; $\lambda_{\text {abs }} 496$ $\mathrm{nm}, \lambda_{\mathrm{em}} 514 \mathrm{~nm}, \Phi_{\mathrm{f}}=0.034$.

$N, N^{\prime}$-(9-Borabicyclo[3.3.1]-non-9-yl)-5-mesityldipyrrin (2d-BN). Following the procedure for $\mathbf{2 a}-\mathbf{M e}_{2}$, the reaction of $\mathbf{8 d}(61 \mathrm{mg}, 0.23 \mathrm{mmol})$ in $\mathrm{CH}_{2} \mathrm{Cl}_{2}(3.0 \mathrm{~mL})$ with TEA $(32 \mu \mathrm{L}, 0.23$ $\mathrm{mmol}$ ) and 9-BBN triflate ( $460 \mu \mathrm{L}$ of a $0.5 \mathrm{M}$ solution in $\mathrm{CH}_{2} \mathrm{Cl}_{2}, 0.46 \mathrm{mmol}$ ) was carried out for $1 \mathrm{~h}$, whereupon TLC analysis (silica, hexanes) indicated that the reaction was complete. The mixture was diluted with $3 \mathrm{~mL}$ of hexanes and chromatographed [silica, hexanes/ $\mathrm{CH}_{2} \mathrm{Cl}_{2}$ (2:1)], providing an orange solid (45 mg, 51\%): $\mathrm{mp} 117-118{ }^{\circ} \mathrm{C} ;{ }^{1} \mathrm{H} \mathrm{NMR} \Delta 0.79$ $0.85(\mathrm{~m}, 2 \mathrm{H}), 1.67-1.79(\mathrm{~m}, 6 \mathrm{H}), 2.04-2.25(\mathrm{~m}, 6 \mathrm{H}), 2.08(\mathrm{~s}, 6 \mathrm{H}), 2.38(\mathrm{~s}, 3 \mathrm{H}), 6.39-6.42(\mathrm{~m}$, 2H), 6.61-6.66 (m, 2H), $6.96(\mathrm{~s}, 2 \mathrm{H}), 8.29-8.32(\mathrm{~m}, 2 \mathrm{H}) ;{ }^{13} \mathrm{C}$ NMR $\Delta$ 20.1, 21.4, 23.2, 25.54 (brs), 31.3, 116.1, 127.4, 128.1, 131.7, 135.7, 136.7, 138.2, 144.9, 146.3; LDMS obsd 381.2, calcd 382.3; FABMS obsd 382.2598, calcd $382.2580\left(\mathrm{C}_{26} \mathrm{H}_{31} \mathrm{BN}_{2}\right) ; \lambda_{\text {abs }} 495 \mathrm{~nm}, \lambda_{\text {em }} 518 \mathrm{~nm}$, $\Phi_{\mathrm{f}}=0.004$.

5-(o-Tolyl)dipyrrin (8c). Following a general procedure, ${ }^{12}$ a solution of $7 \mathbf{c}(2.50 \mathrm{~g}, 10.6$ $\mathrm{mmol})$ in THF $(50 \mathrm{~mL})$ was treated dropwise with a solution of DDQ $(2.41 \mathrm{~g}, 10.6 \mathrm{mmol})$ in THF $(25 \mathrm{~mL})$ at room temperature. After stirring for $1 \mathrm{~h}$, TLC analysis showed incomplete oxidation. Therefore, a solution of DDQ $(0.482 \mathrm{~g}, 2.12 \mathrm{mmol})$ in THF $(2 \mathrm{~mL})$ was added and the reaction mixture was stirred for another $20 \mathrm{~min}$. The solvent was removed and the resulting 
crude was chromatographed [silica, $\mathrm{CH}_{2} \mathrm{Cl}_{2} / \mathrm{MeOH}$ (98:2)], affording a brown-yellowish solid (2.09 g, 84\%): $\mathrm{mp} 79-80{ }^{\circ} \mathrm{C} ;{ }^{1} \mathrm{H}$ NMR $\Delta 2.19(\mathrm{~s}, 3 \mathrm{H}), 6.42(\mathrm{dd}, J=4.4 \mathrm{~Hz}, J=1.6 \mathrm{~Hz}, 2 \mathrm{H})$, 6.49-6.51 (m, 2H), 7.26-7.30 (m, 3H), 7.36-7.42 (m, 2H), 7.80-7.84 (m, 2H); ${ }^{13} \mathrm{C}$ NMR $\Delta$ 19.8, 117.4, 124.9, 128.8, 129.9, 130.2, 131.2, 136.7, 144.1, 154.4; LDMS obsd 234.8; calcd 234.3; FABMS obsd 235.1234, calcd $235.1235\left[(\mathrm{M}+\mathrm{H})^{+} ; \mathrm{M}=\mathrm{C}_{16} \mathrm{H}_{14} \mathrm{~N}_{2}\right] ; \lambda_{\text {abs }} 432 \mathrm{~nm}$.

\section{Photophysical Characterization.}

Static Absorption and Emission Spectroscopy. Static absorption (Cary 100) and fluorescence (Spex Tau2) measurements were performed as described previously. ${ }^{18}$ For emission studies, non-deaerated samples with an absorbance $\leq 0.15$ (typically $0.3-0.8 \mu \mathrm{M}$ ) at $\lambda_{\text {exc }}$ were employed. Emission quantum yields were measured relative to fluorescein in $0.1 \mathrm{M}$ $\mathrm{NaOH}\left(\Phi_{\mathrm{f}}=0.92\right)^{19}>$ and were corrected for solvent refractive index.

Time-Resolved Fluorescence Spectroscopy. Fluorescence lifetimes were obtained on samples that had concentrations of $0.5-10 \mu \mathrm{M}$ and were deaerated by bubbling with $\mathrm{N}_{2}$. Lifetimes were determined by the fluorescence modulation (phase shift) technique using a Spex Tau2 spectrofluorometer. Samples were excited at various wavelengths and detected through appropriate colored glass filters. Modulation frequencies from $20-300 \mathrm{MHz}$ were utilized and both the fluorescence phase shift and modulation amplitude were analyzed.

Time-Resolved Absorption Spectroscopy. Transient absorption data were acquired as described previously. ${ }^{18}$ Samples $(\sim 10 \mu \mathrm{M})$ in $2 \mathrm{~mm}$ pathlength cuvettes at room temperature were excited at $10 \mathrm{~Hz}$ with $\sim 130 \mathrm{fs}, 5-30 \mu \mathrm{J}$ pulses at the appropriate wavelength and probed with white-light probe pulses of comparable duration. Spectra shown at $<1 \mathrm{ps}$ were constructed from spectra at closely spaced time intervals to account for the time-dispersion of wavelengths in the white-light probe pulse. Kinetic data were fit to functions consisting of one to three exponentials (convoluted with an instrument response) plus a constant asymptote.

\section{Theory}

Ground-state potential energy surfaces were generated by scanning the aryl dihedral angle and optimizing the remaining degrees of freedom using density functional methods and redundant internal coordinates. We selected the B3LYP hybrid functional of Becke, Lee, Yang, and Parr 20,21 and used a 6-31G(d) basis set. This combination provides a near optimum combination of accuracy and computational efficiency. ${ }^{22}$ The lowest excited triplet state surfaces were generated by using the identical procedures, and the triplet state geometry was then used as the guess geometry for the excited singlet state optimizations. The lowest singlet state geometries were calculated by using gradients generated within full single excitation configuration interaction with inner shell orbitals (e.g., carbon 1s) frozen. The resulting energies were then adjusted for correlation by using double $\mathrm{CI}$ as provided by a windowed [32 (filled)+32(open)] symmetry-adapted cluster configuration interaction (SACCI) calculation. ${ }^{23} \mathrm{The} \operatorname{Opt}(\mathrm{CIS})+\Delta \mathrm{E}(\mathrm{CID} / \mathrm{SACCI})$ approximation can be justified on the basis of two observations. First, as we will demonstrate later, the lowest singlet state is an ionic state dominated by singly excited configurations $(>80 \%)$. Second, test calculations using SACCI with single and double CI indicate that single CI optimization generates a geometry that is within a few percent of that generated using single and double CI in terms of redundant internal coordinates when the aryl group dihedral angle is fixed. Nevertheless, double CI plays an important role in the energy correlation of the excited singlet state when the aryl ring approaches planarity with respect to the boron-dipyrrin framework.

Excited-state spectroscopic properties were calculated by using MNDO-PSDCI ${ }^{24,25}$ and $\mathrm{SACCI}^{23}$ methods with partial single and double $\mathrm{CI}$ assuming vacuum conditions. Test calculations using full CISD on high symmetry molecules indicated that the states calculated 
using restricted basis set had converged to within $0.1 \mathrm{eV}$ of the full CISD values. The MNDOPSDCI and SACCI methods generate comparable energies and properties for the lowest four excited singlet states, but these methods diverge for the higher energy transitions. We report here only the results for the SACCI calculations, which we consider to be more accurate and reliable. The SACCI calculations used the Huzinaga-Dunning D95 double zeta basis set, which has been shown to be optimal for spectroscopic calculations. 23

The $a b$-initio and density functional calculations were carried out using Gaussian-03 Linux Revision B05 26 with Linda as the parallel resource software running under Red Hat Linux 9.0 (Red Hat, Inc., Raleigh, NC). The hardware environment was a 16-node (32 processors) Beowulf cluster manufactured by Western Scientific (San Diego, CA). Each node is composed of two $1266 \mathrm{MHz}$ Intel Pentium III processors, 1 GB of DDR RAM, and a single $34 \mathrm{~GB}$ SEAGATE hard drive for local data buffering. The nodes are networked using fast Ethernet (Ethernet Pro 100). The semiempirical MNDO-PSDCI and Gaussian-03 survey calculations were run on a dual processor $1250 \mathrm{MHz}$ Macintosh G5.

\title{
Supplementary Material
}

Refer to Web version on PubMed Central for supplementary material.

\begin{abstract}
Acknowledgement
This research was supported by grants from the Department of Energy (JSL, DFB, and DH) and the National Institutes of Health (GM-38401 to W.R.S). Mass spectra were obtained at the Mass Spectrometry Laboratory for Biotechnology at North Carolina State University. Partial funding for the NCSU Facility was obtained from the North Carolina Biotechnology Center and the NSF.
\end{abstract}

\section{References}

(1). Treibs A, Kreuzer F-H. Liebigs Ann. Chem 1968;718:208-223.

(2). (a) Kim H, Burghart A, Welch MB, Reibenspies J, Burgess K. Chem. Commun 1999:1889-1890. (b) Burghart A, Kim H, Welch MB, Thoresen LH, Reibenspies J, Burgess K. J. Org. Chem 1999;64:7813-7819. (c) Chen J, Burghart A, Derecskei-Kovacs A, Burgess K. J. Org. Chem 2000;65:2900-2906. [PubMed: 10814176] (d) Burghart A, Thoresen LH, Chen J, Burgess K, Bergström F, Johansson LB-Å. Chem. Commun 2000:2203-2204.

(3). Lee C-H, Lindsey JS. Tetrahedron 1994;50:11427-11440.

(4). Littler BJ, Miller MA, Hung C-H, Wagner RW, O'Shea DF, Boyle PD, Lindsey JS. J. Org. Chem 1999;64:1391-1396.

(5). Laha JK, Dhanalekshmi S, Taniguchi M, Ambroise A, Lindsey JS. Org. Process Res. Dev 2003;7:799-812.

(6). Brückner C, Karunaratne V, Rettig SJ, Dolphin D. Can. J. Chem 1996;74:2182-2193.

(7). Wagner RW, Lindsey JS. J. Am. Chem. Soc 1994;116:9759-9760.

(8). (a) Wagner RW, Lindsey JS. Pure Appl. Chem 1996;68:1373-1380. (b) Wagner RW, Lindsey JS. Pure Appl. Chem 1998;70(8):i.

(9). Ambroise A, Kirmaier C, Wagner RW, Loewe RS, Bocian DF, Holten D, Lindsey JS. J. Org. Chem 2002;67:3811-3826. [PubMed: 12027698]

(10). (a) Wagner RW, Lindsey JS, Seth J, Palaniappan V, Bocian DF. J. Am. Chem. Soc 1996;118:39963997. (b) Ambroise A, Wagner RW, Rao PD, Riggs JA, Hascoat P, Diers JR, Seth J, Lammi RK, Bocian DF, Holten D, Lindsey JS. Chem. Mater 2001;13:1023-1034.

(11). Li F, Yang SI, Ciringh Y, Seth J, Martin CH III, Singh DL, Kim D, Birge RR, Bocian DF, Holten D, Lindsey JS. J. Am. Chem. Soc 1998;120:10001-10017.

(12). Yu L, Muthukumaran K, Sazanovich IV, Kirmaier C, Hindin E, Diers JR, Boyle PD, Bocian DF, Holten D, Lindsey JS. Inorg. Chem 2003;42:6629-6647. [PubMed: 14552615]

(13). Sazanovich IV, Kirmaier C, Hindin E, Yu L, Bocian DF, Lindsey JS, Holten D. J. Am. Chem. Soc 2004;126:2664-2665. [PubMed: 14995159] 
(14). Jones G, Kumar S, Klueva O, Pacheco D. J. Phys. Chem. A 2003;107:8429-8434.

(15). Arbeloa FL, Arbeloa TL, Arbeloa IL. J. Photochem. Photobiol. A 1999;121:177-182.

(16). Karolin J, Johansson LB-A, Strandberg L, Ny T. J. Am. Chem. Soc 1994;116:7801-7806.

(17). Johnson ID, Kang HC, Haugland RP. Anal. Biochem 1991;198:228-237. [PubMed: 1799206]

(18). (a) Prathapan S, Yang SI, Seth J, Miller MA, Bocian DF, Holten D, Lindsey JS. J. Phys. Chem. B 2001;105:8237-8248. (b) Yang SI, Li J, Cho HS, Kim D, Bocian DF, Holten D, Lindsey JS. J. Mater. Chem 2000;10:283-296.

(19). Demas, JN. (Measurement of Photoluminescence). In: Mielenz, KD., editor. Optical Radiation Measurements. V3. Academic Press; New York: 1982. p. 195-248.

(20). (a) Becke AD. Phys. Rev. A 1988;38:3098-3100. [PubMed: 9900728] (b) Becke AD. J. Chem. Phys 1992;97:9173-9177. (c) Becke AD. J. Chem. Phys 1992;96:2155-2160. (d) Becke AD. J. Chem. Phys 1993;98:5648-5652. (e) Becke AD. J. Chem. Phys 1996;104:1040-1046.

(21). Miehlich B, Savin A, Stoll H, Preuss H. Chem. Phys. Lett 1989;157:200-206.

(22). Foresman, JB.; Frisch, E. Exploring chemistry with electronic structure methods. Gaussian Inc.; 2000. 2nd ed.

(23). (a) Miyahara T, Tokita Y, Nakatsuji H. J. Phys. Chem. B 2001;105:7341-7352. (b) Miyahara T, Nakatsuji H, Hasegawa J, Osuka A, Aratani N, Tsuda A. J. Chem. Phys 2002;117:11196-11206. (c) Nakajima T, Nakatsuji H. Chem. Phys 1999;242:177-193. (d) Nakatsuji H. Chem. Phys. Lett 1978;59:362-364. (e) Nakatsuji H, Hirao K. J. Chem. Phys 1978;68:2053-2065. (f) Nakatsuji H. Chem. Phys. Lett 1991;177:331-337.

(24). Martin CH, Birge RR. J. Phys. Chem. A 1998;102:852-860.

(25). Ren L, Martin CH, Wise KJ, Gillespie NB, Luecke H, Lanyi JK, Spudich JL, Birge RR. Biochemistry 2001;40:13906-13914. [PubMed: 11705380]

(26). Frisch, MJ.; Trucks, GW.; Schlegel, HB.; Scuseria, GE.; Robb, MA.; Cheeseman, JR.; Montgomery, JA., Jr.; Vreven, T.; Kudin, KN.; Burant, JC.; Millam, JM.; Iyengar, SS.; Tomasi, J.; Barone, V.; Mennucci, B.; Cossi, M.; Scalmani, G.; Rega, N.; Petersson, GA.; Nakatsuji, H.; Hada, M.; Ehara, M.; Toyota, K.; Fukuda, R.; Hasegawa, J.; Ishida, M.; Nakajima, T.; Honda, Y.; Kitao, O.; Nakai, H.; Klene, M.; Li, X.; Knox, JE.; Hratchian, HP.; Cross, JB.; Bakken, V.; Adamo, C.; Jaramillo, J.; Gomperts, R.; Stratmann, RE.; Yazyev, O.; Austin, AJ.; Cammi, R.; Pomelli, C.; Ochterski, JW.; Ayala, PY.; Morokuma, K.; Voth, GA.; Salvador, P.; Dannenberg, JJ.; Zakrzewski, VG.; Dapprich, S.; Daniels, AD.; Strain, MC.; Farkas, O.; Malick, DK.; Rabuck, AD.; Raghavachari, K.; Foresman, JB.; Ortiz, JV.; Cui, Q.; Baboul, AG.; Clifford, S.; Cioslowski, J.; Stefanov, BB.; Liu, G.; Liashenko, A.; Piskorz, P.; Komaromi, I.; Martin, RL.; Fox, DJ.; Keith, T.; Al-Laham, MA.; Peng, CY.; Nanayakkara, A.; Challacombe, M.; Gill, PMW.; Johnson, B.; Chen, W.; Wong, MW.; Gonzalez, C.; Pople, JA. Gaussian, Inc.; Wallingford CT: 2004. 
<smiles></smiles>

$\beta$-substituted boron-dipyrrin dyes<smiles></smiles>

meso- (5) substituted boron-dipyrrin dyes

chart 1 

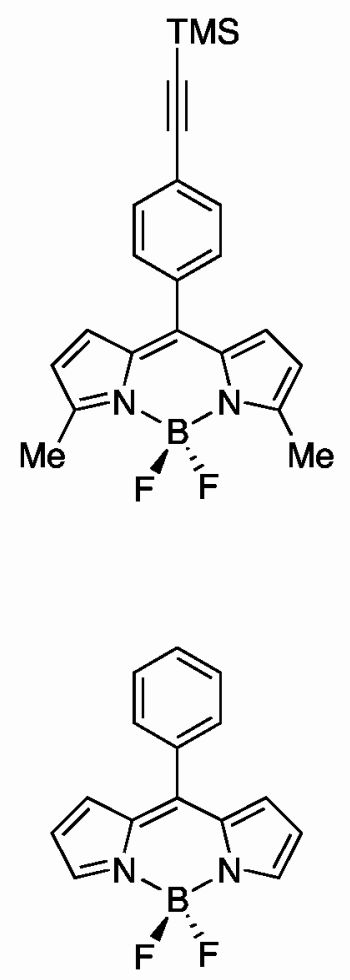

2a

chart 2 

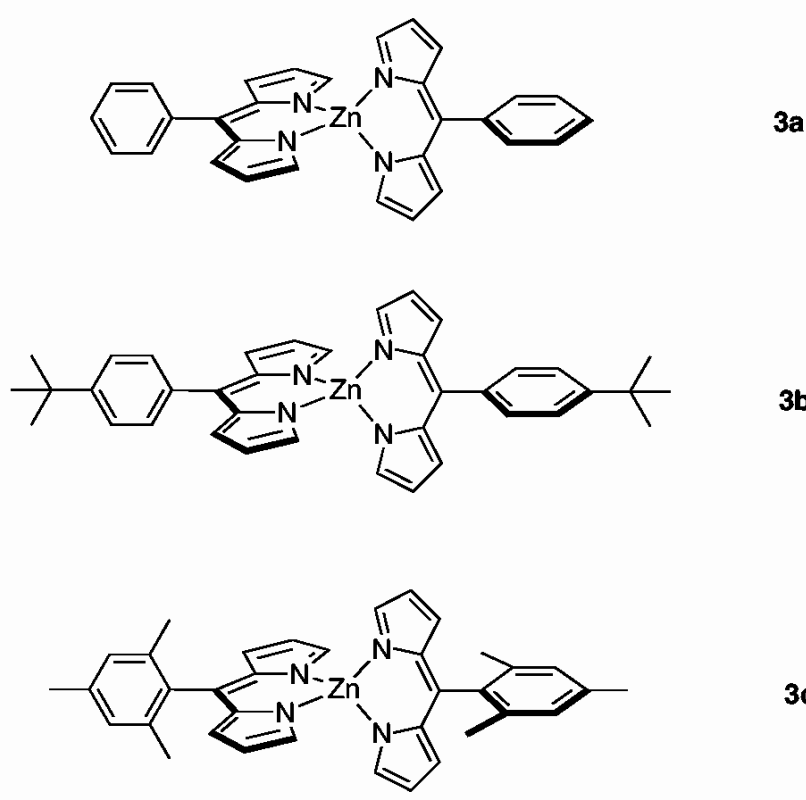

chart 3 


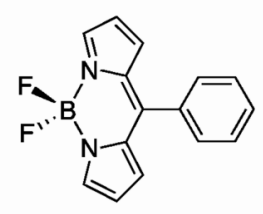

$2 a$

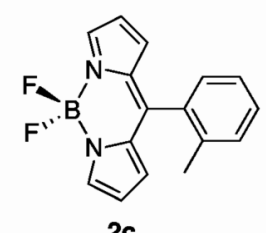

2c
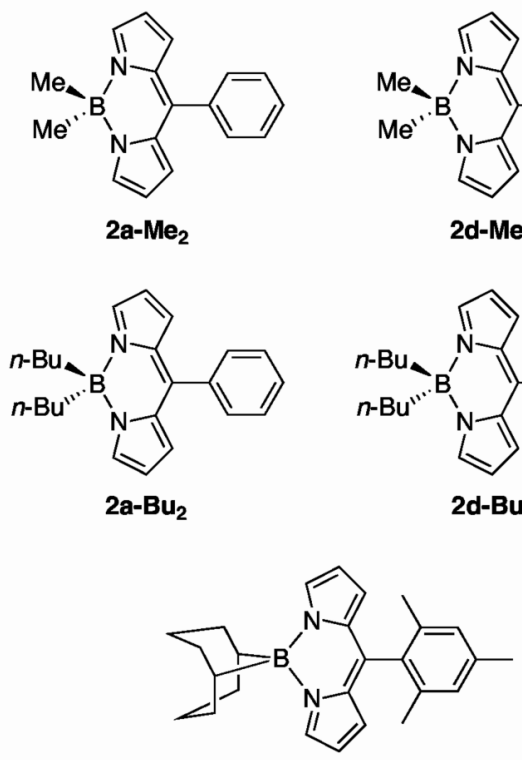

2d-BN

2d
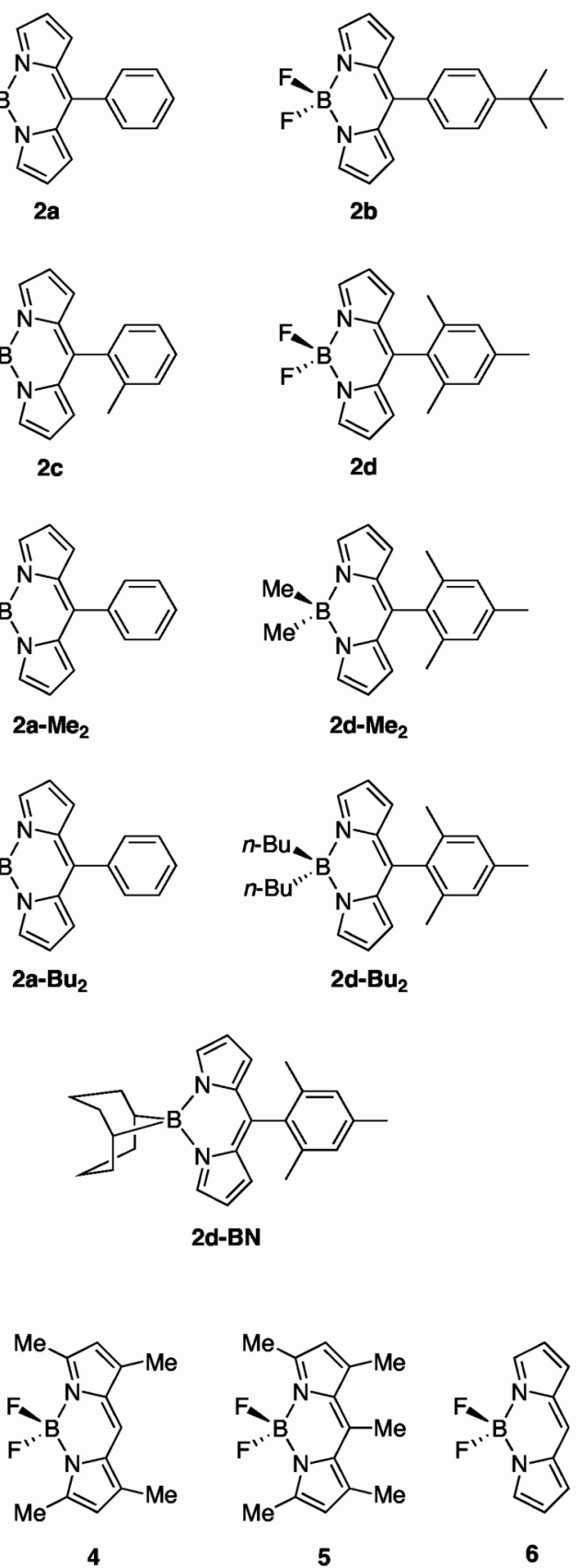

chart 4 
<smiles>Cc1ccccc1C(c1ccc[nH]1)c1ccc[nH]1</smiles>

7c
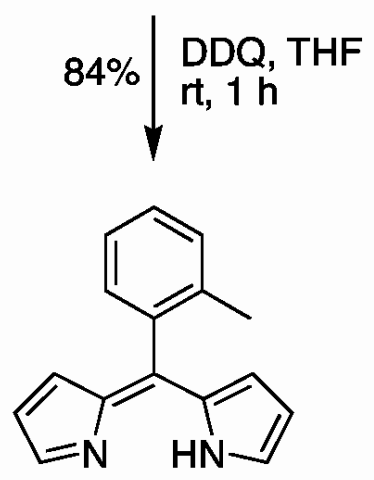

8c

scheme 1 


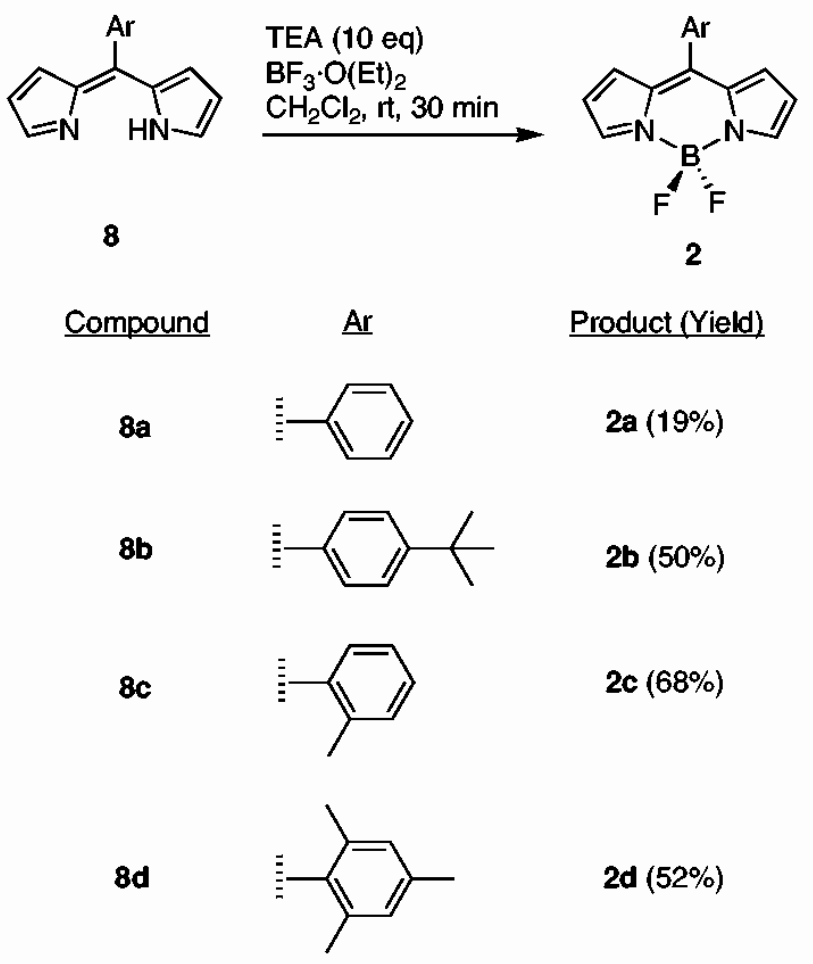

scheme 2 

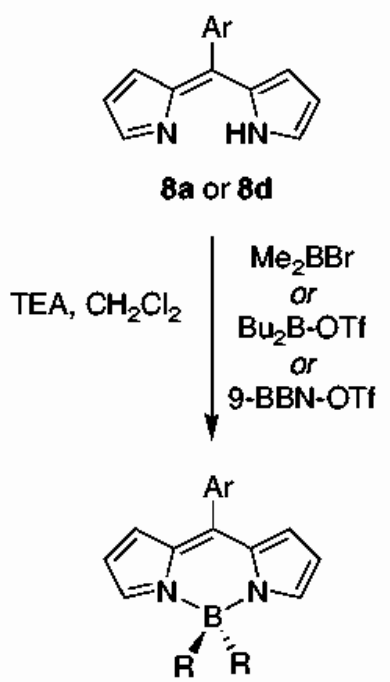

$2 \mathrm{a}-\mathrm{Me}_{2} ; \mathrm{Ar}=$ phenyl, $\mathbf{R}=$ methyl $(89 \%)$

$2 \mathrm{a}-\mathrm{Bu}_{\mathbf{2}} ; \mathrm{Ar}=$ phenyl, $\mathbf{R}=$ butyl $(26 \%)$

2d-Me $;$; $\mathrm{Ar}=$ mesityl, $\mathrm{R}=$ methyl $(87 \%)$

2d-Bu $; \mathrm{Ar}=$ mesityl, $\mathbf{R}=$ butyl $(19 \%)$

2d-BN; $\mathrm{Ar}=$ mesityl, $\mathbf{R}, \mathbf{R}=$ bicylononyl $(51 \%)$

scheme 3 


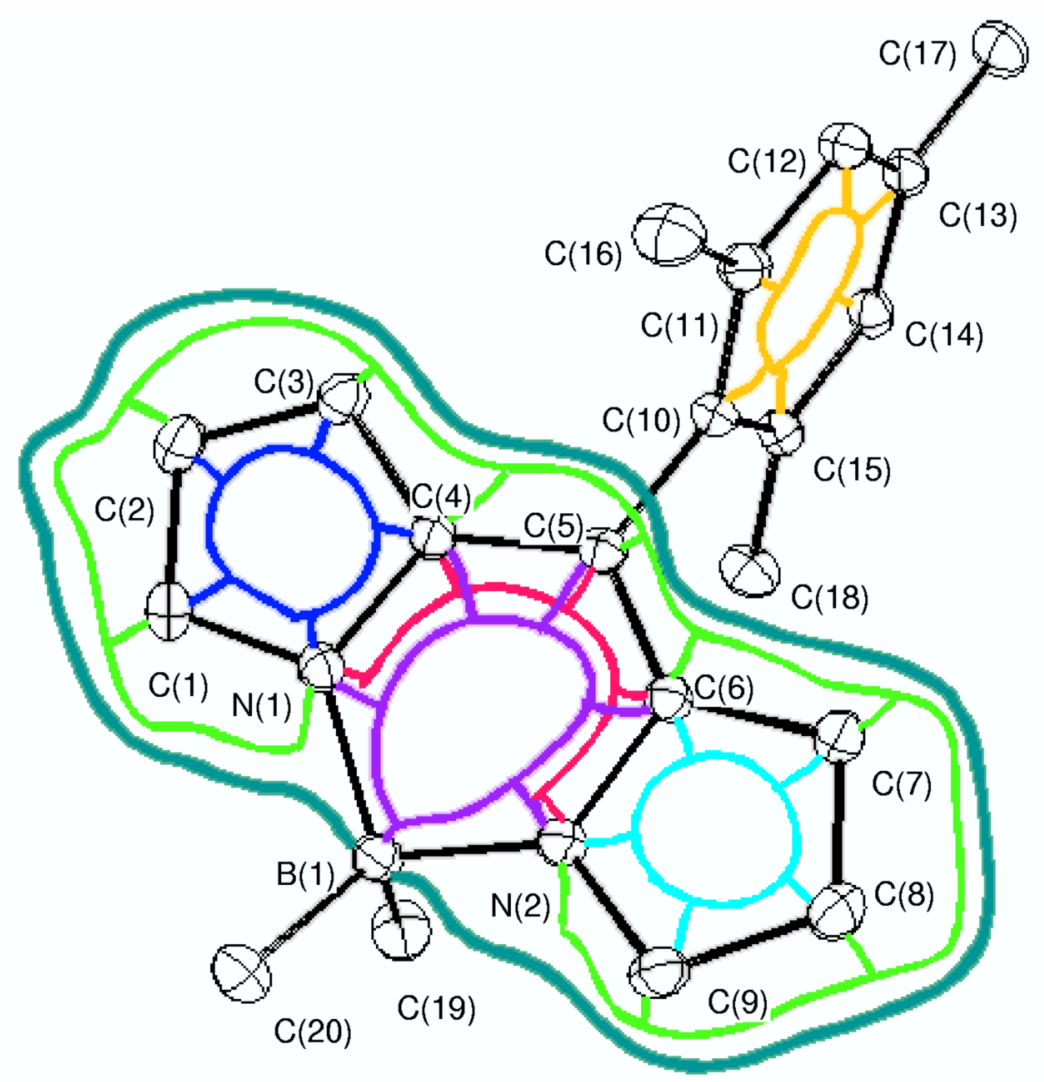

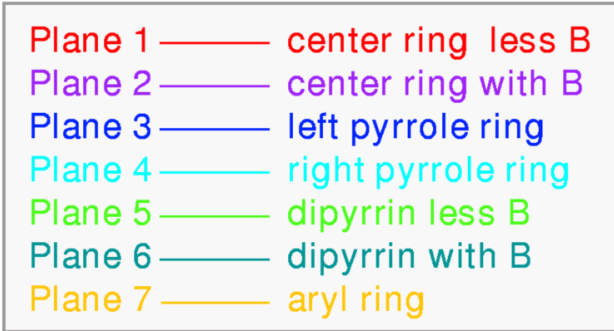

Figure 1.

Basic architecture and atom numbering of a 5-aryl-substituted dipyrrin. Planes encompassing a number of architectural features are indicated by the colored lines. The dihedral angles between these planes are given in Table 1. 


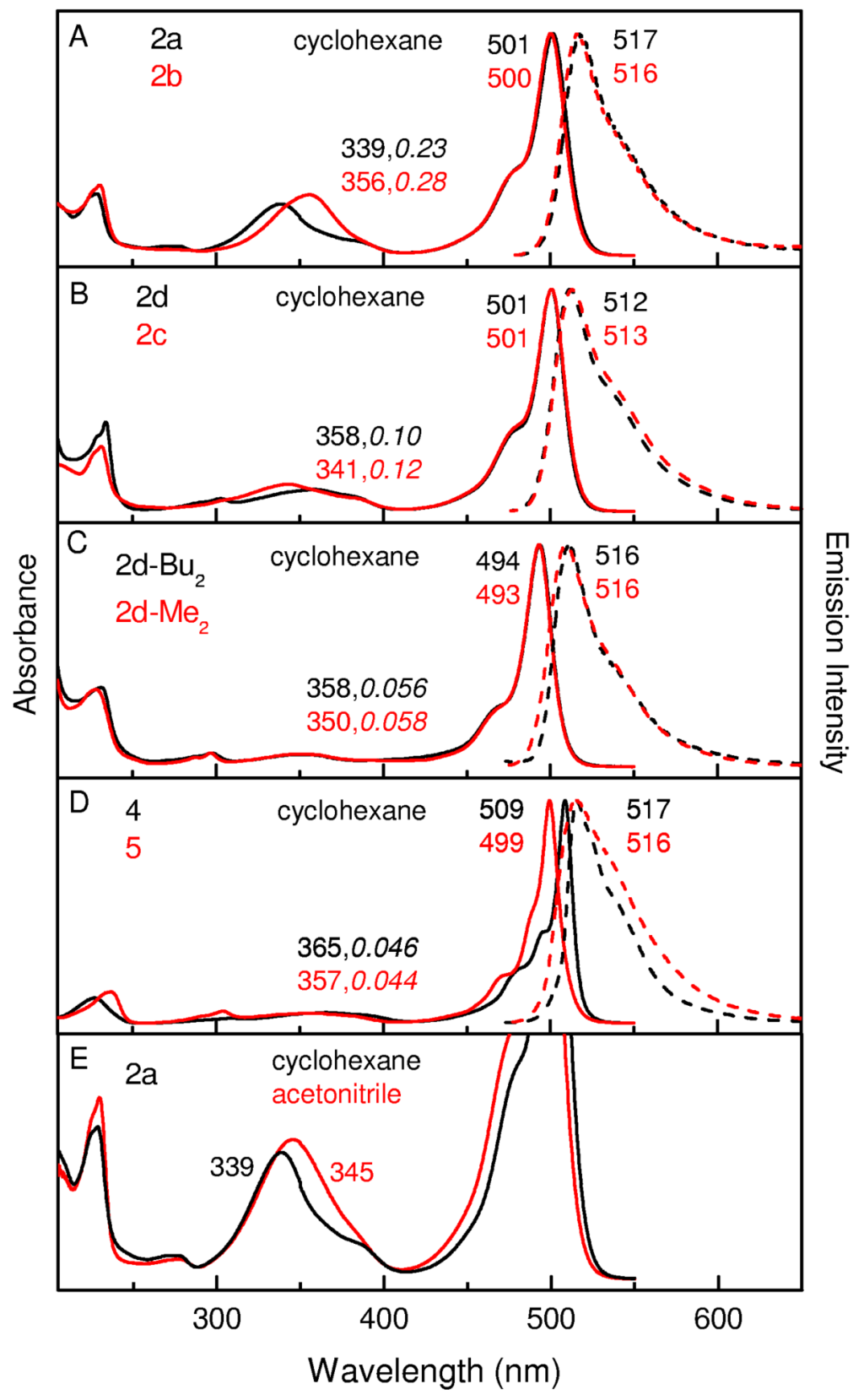

Figure 2.

Room temperature electronic absorption spectra (solid) and fluorescence spectra(dashed) of boron-dipyrrin dyes. The peak wavelengths are indicated. The second value indicated for the absorption in the 300-400 nm region in panels A-E is the peak amplitude compared to the maximum of the lowest-energy transition in the $490-510 \mathrm{~nm}$ region. The fluorescence spectra were acquired using an excitation wavelength of $450-460 \mathrm{~nm}$. The spectra in panel $\mathrm{E}$ are vertically enhanced $\sim 3$-fold from the other panels to emphasize the solvent dependence of the spectra in the $300-400 \mathrm{~nm}$ region. 


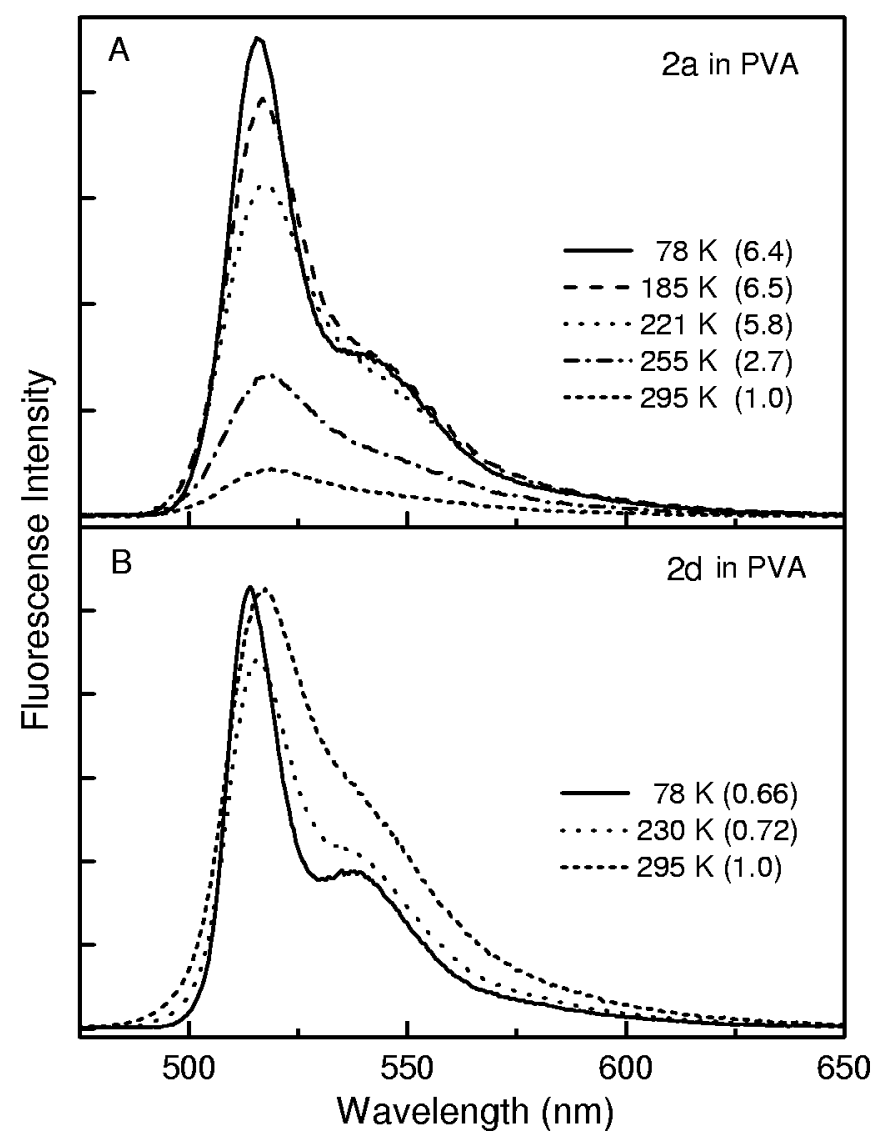

Figure 3.

Temperature dependence of the fluorescence for $\mathbf{2 a}(\mathrm{A})$ and $\mathbf{2 d}(\mathrm{B})$ in PVA. The samples were excited at 455 or $450 \mathrm{~nm}$, respectively. 

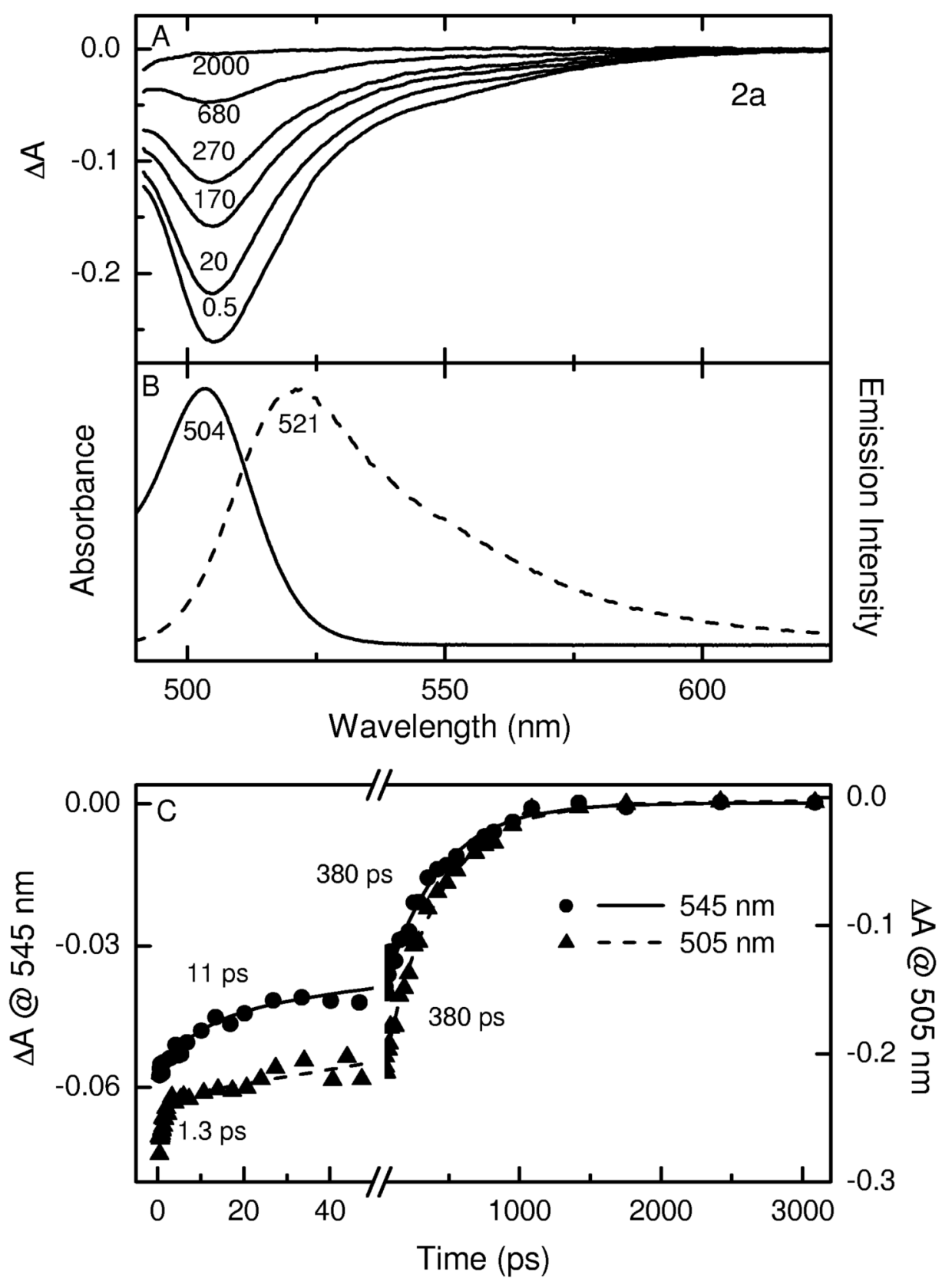

Figure 4.

Time-resolved absorption difference spectra for $\mathbf{2 a}$ in toluene at room temperature using $\sim 100$ fs excitation flashes at $483 \mathrm{~nm}$ (A); the time (in picoseconds) for each spectrum is indicated. Absorption (solid) and fluorescence (dashed) spectra for this compound (B). Representative kinetic traces and dual-exponential fits at $505 \mathrm{~nm}$ (ground-state absorption bleaching decay) and $545 \mathrm{~nm}$ (stimulated emission decay) are shown in panel C. 

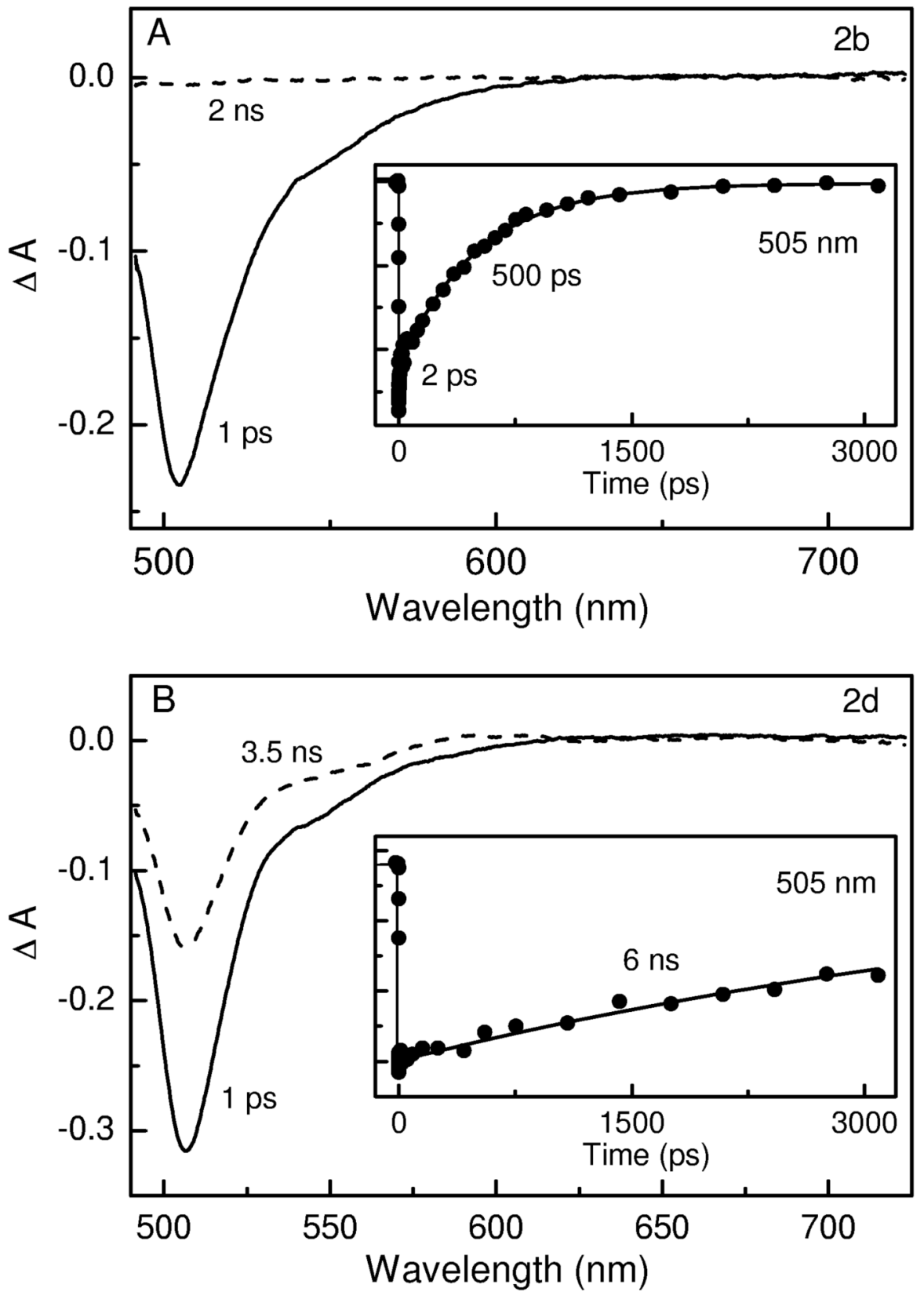

Figure 5.

Time-resolved absorption and kinetic data (at $505 \mathrm{~nm}$ ) for $\mathbf{2 b}(\mathrm{A})$ and $\mathbf{2 a}$ (B). Other conditions are as in Figure 4. 


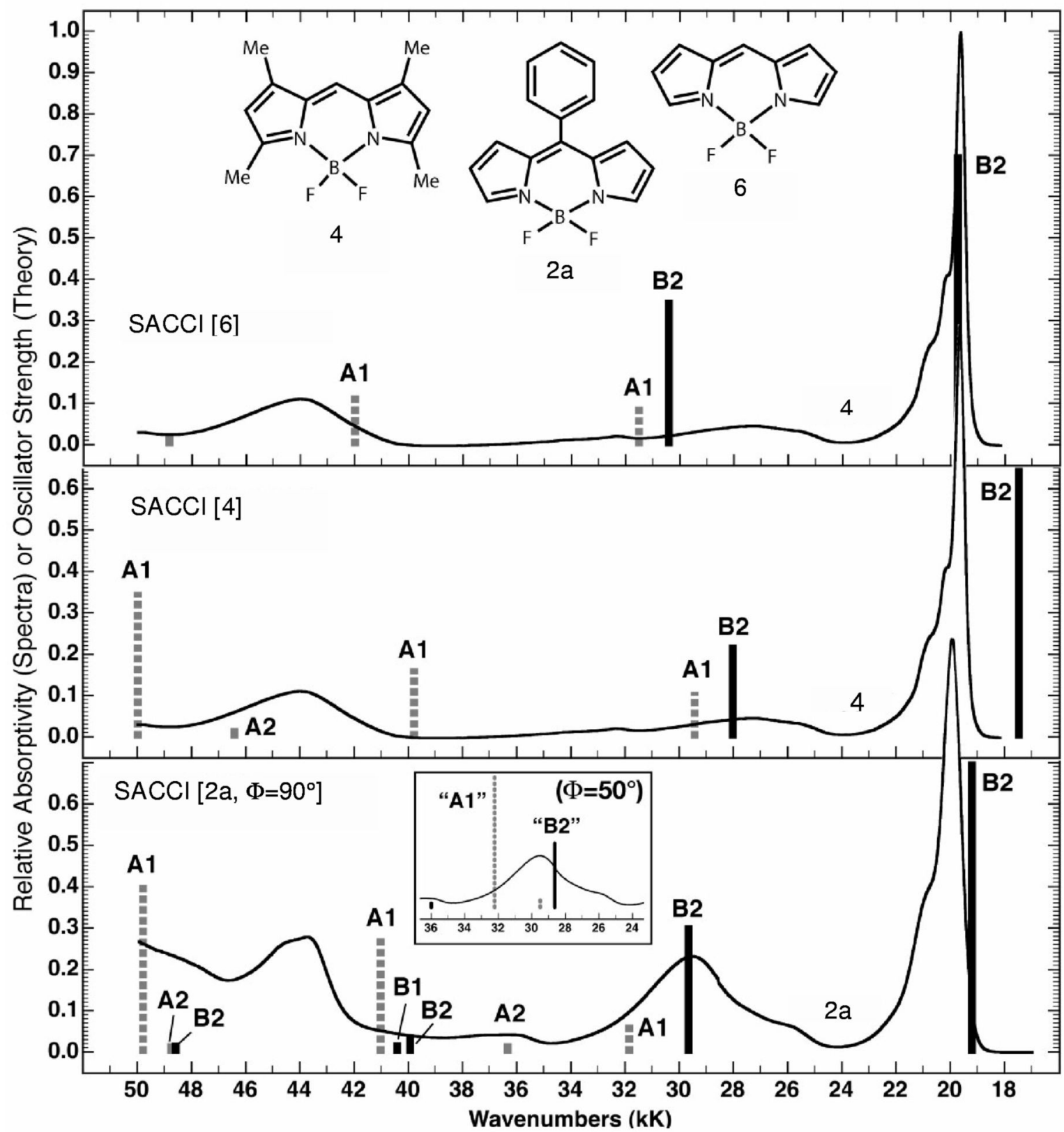

Figure 6.

Comparison of the absorption spectra of $\mathbf{4}$ (top two spectra) and $\mathbf{2 a}$ (lower spectrum) with the calculated singlet state transition energies for $\mathbf{6}, \mathbf{4}$ and $\mathbf{2 a}$ with the phenyl group rotated orthogonal to the boron-dipyrrin plane. The ground-state geometries were optimized using B3LYP/6-31G(d) methods and the spectroscopic properties were calculated using SACCI methods with single and double CI (see text). The heights of the bands are proportional to the oscillator strengths of the bands. Forbidden or very weak bands are assigned an oscillator strength of 0.04 so that the band is visible. 


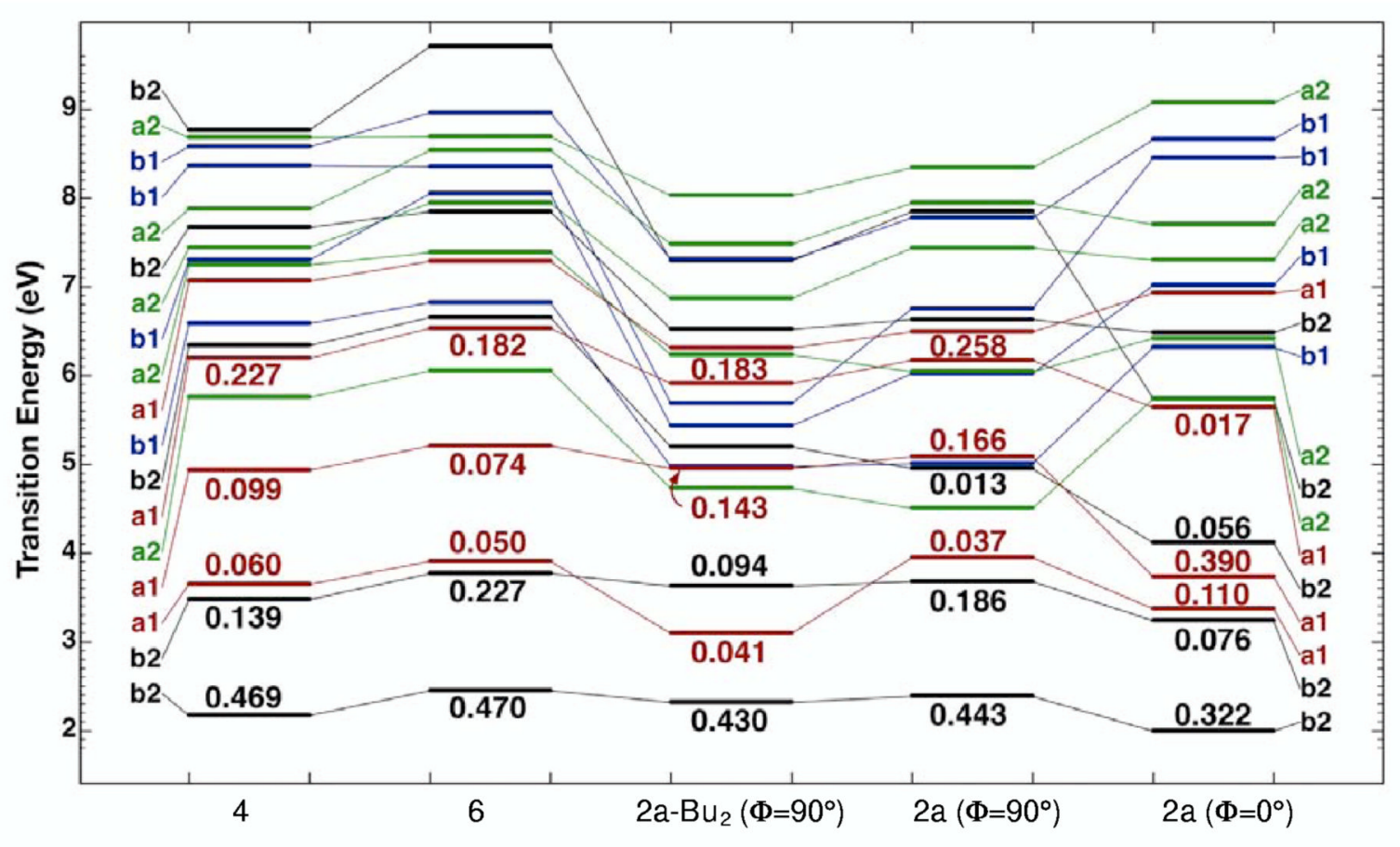

Figure 7.

Comparison of the excited-state level ordering in $\mathbf{6 ,}, \mathbf{4}, \mathbf{2} \mathbf{a}-\mathbf{B} \mathbf{u}_{2}$ and $\mathbf{2 a}$ on the basis of SACCI methods including single and double CI (see text). The oscillator strengths for low-lying strongly allowed states are shown above or below the horizontal band representing the transition energy. The calculations for $\mathbf{2 a}$ were carried out for two geometries of the phenyl ring: orthogonal $\left(\Phi=90^{\circ}\right)$ and parallel $\left(\Phi=0^{\circ}\right)$ to the boron-dipyrrin plane, for which the symmetry labels are approximate. The calculation for $\mathbf{2} \mathbf{a}-\mathbf{B} \mathbf{u}_{2}$ was carried out for an orthogonal phenyl group $\left(\Phi=90^{\circ}\right)$. Other details are as in Figure 6 . Note that a states are symmetry forbidden $(f=0.0)$ and that all of the low-lying $b_{1}$ states are very weak $(f<0.01)$. 


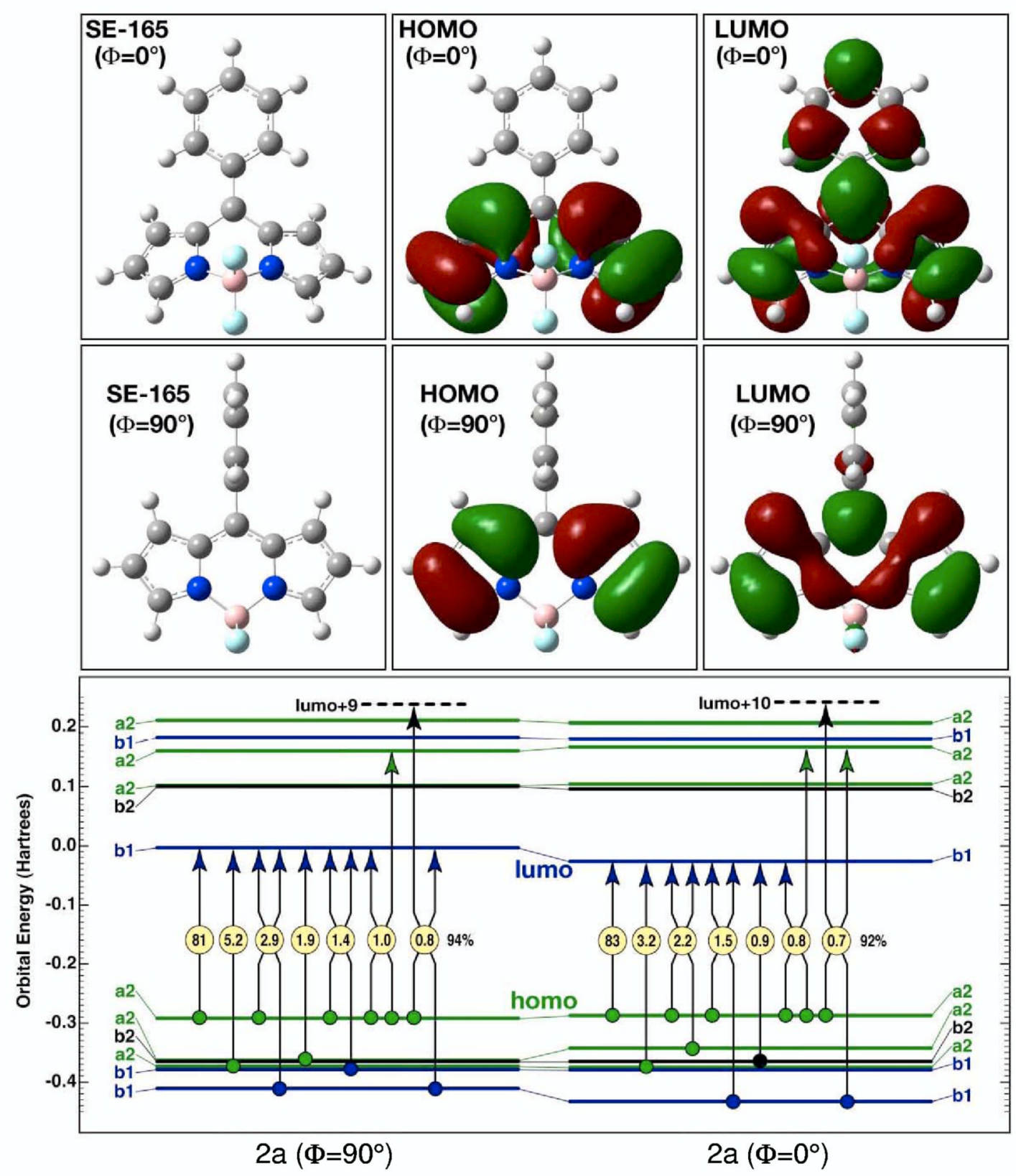

Figure 8.

The primary configurational properties of the lowest-lying $\mathrm{B}_{2}$ singlet states of $\mathbf{2 a}$ for two geometries of the phenyl ring: orthogonal to the boron-dipyrrin plane $\left(\Phi=90^{\circ}\right.$, left $)$ and parallel to the dipyrrin plane $\left(\Phi=0^{\circ}\right.$, right). The lowest excited singlet state is highly ionic in character and is well described as a simple $\operatorname{HOMO}\left(\mathrm{a}_{2}\right) \rightarrow$ LUMO $\left(\mathrm{b}_{1}\right)$ one-electron excitation. The nature of the highest occupied molecular orbital (HOMO, $\mathrm{a}_{2}$ or $\mathrm{a}_{2}$-like symmetry) is relatively invariant in both character and energy to phenyl rotation. In contrast, the lowest unoccupied molecular orbital (LUMO, $b_{1}$ or $b_{1}$-like symmetry) extends into the phenyl group upon rotation and is highly stabilized by the increased extent of the pi-electron system when the phenyl group is parallel to the boron-dipyrrin plane $\left(\Phi=0^{\circ}\right)$. Stabilization of the LUMO is the dominant 
electronic driving force for phenyl group relaxation towards planarity in the $S_{1}$ excited singlet state. 

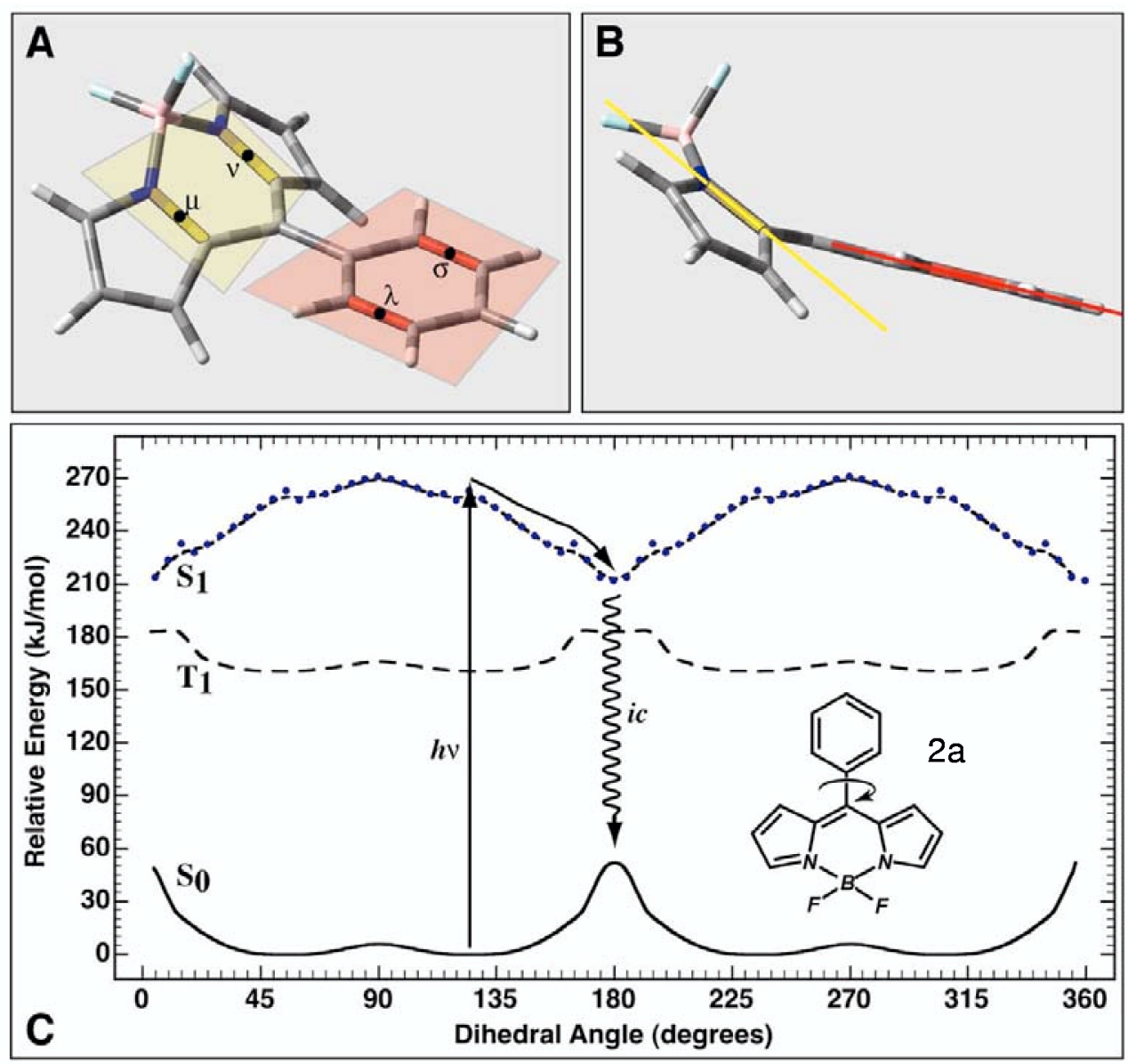

Figure 9.

The ground, first excited triplet state and first excited singlet state potential energy surfaces of $\mathbf{2 a}$ as a function of phenyl group rotation is shown in $\mathrm{C}$. As the phenyl group rotates into the plane of the boron-dipyrrin framework, repulsions between the hydrogen atoms on both groups force a puckering as shown in $\mathrm{A}$ and $\mathrm{B}$. This distortion requires that we define the angle of rotation with reference to the rotation of the yellow and red planes. In practice, the dihedral angle is calculated in terms of the bond centroids $v, v, \lambda$ and $\sigma$ as shown in insert $\mathrm{A}$. The alignment shown in $\mathrm{B}$ represents a dihedral angle of $0^{\circ}$ (which because of symmetry is identical to $180^{\circ}$ or $360^{\circ}$ ). The ground and triplet state surfaces were calculated using B3LYP/6-31G (d) methods. The excited singlet state surface was calculated using full single CI minimization with a correlative correction calculated by using SACCI doubles (see text). 


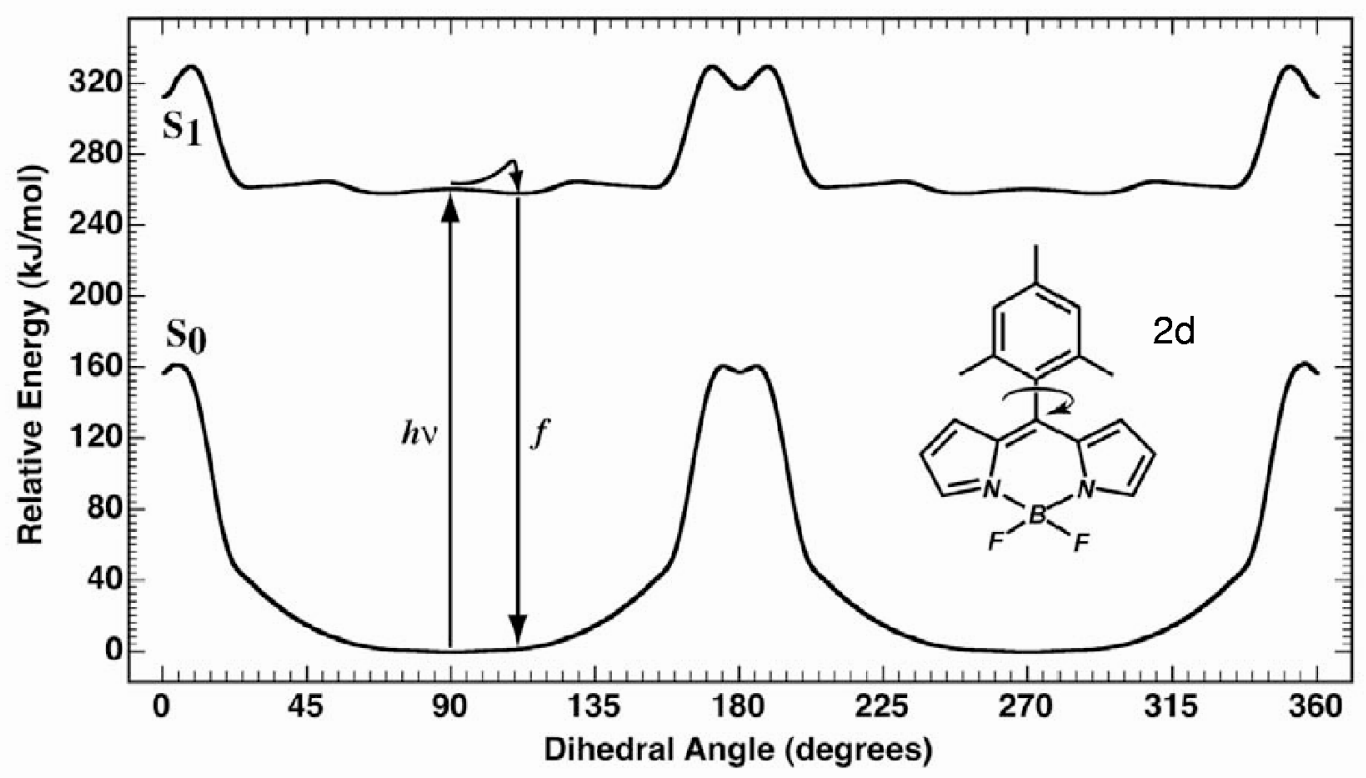

Figure 10.

The ground and first excited singlet state potential energy surfaces of $\mathbf{2 d}$ as a function of mesityl group rotation. Details are as in Figure 9. 
Table 1.

Dihedral angles between planes in boron-dipyrrin dyes.

\begin{tabular}{|c|c|c|c|c|c|c|}
\hline plane & $\begin{array}{c}\text { 5- } \\
\text { group: B- } \\
\text { group: }\end{array}$ & 2a phenyl F & $\begin{array}{c}\text { 2bt-Bu- } \\
\text { phenyl F }\end{array}$ & 2d mesityl F & 2co-tolyl F & $\begin{array}{c}\text { 2d-Me } \mathrm{Mesityl}_{2} \\
\mathrm{Me}\end{array}$ \\
\hline 1,2 & & 0.0 & 2.9 & 0.7 & 0.9 & 5.7 \\
\hline 1,3 & & 1.9 & 7.0 & 1.7 & 2.5 & 7.1 \\
\hline 2,3 & & 1.9 & 6.4 & 1.0 & 2.9 & 8.9 \\
\hline 1,4 & & 1.9 & 1.0 & 2.7 & 2.9 & 8.4 \\
\hline 2,4 & & 1.9 & 3.9 & 3.1 & 2.8 & 8.8 \\
\hline 3,4 & & 2.6 & 7.7 & 3.4 & 5.4 & 15.3 \\
\hline 1,5 & & 0.7 & 1.6 & 0.7 & 0.3 & 0.3 \\
\hline 2,5 & & 0.7 & 3.2 & 1.0 & 1.1 & 5.7 \\
\hline 3,5 & & $\begin{array}{l}0.1 \\
1.5\end{array}$ & 5.5 & 1.6 & 2.5 & 7.4 \\
\hline 4,5 & & 1.5 & 2.2 & 0.1 & 2.9 & 8.2 \\
\hline 1,6 & & 0.7 & 2.7 & 0.9 & 0.7 & 4.3 \\
\hline 2,6 & & 0.7 & 1.7 & 0.8 & 0.3 & 1.4 \\
\hline 3,6 & & 1.5 & 4.9 & 1.1 & 2.8 & 8.4 \\
\hline 4,6 & & 1.5 & 3.7 & 2.5 & 2.7 & 8.1 \\
\hline 5,6 & & 0.0 & 2.0 & 0.5 & 0.9 & 4.3 \\
\hline 1,7 & & 60.8 & 50.0 & 75.4 & 84.8 & 84.4 \\
\hline 2,7 & & 60.8 & 50.2 & 75.3 & 84.8 & 84.7 \\
\hline 3,7 & & 2.1 & 56.6 & 75.9 & 2.3 & 77.3 \\
\hline 4,7 & & 62.1 & 49.6 & 77.9 & 7.6 & 92.6 \\
\hline 5,7 & & 61.4 & 51.6 & 76.1 & 84.9 & 84.6 \\
\hline 6,7 & & 61.4 & 51.7 & 71.6 & 84.9 & 84.9 \\
\hline
\end{tabular}

${ }^{a}$ Substituent group at the 5-position.

${ }^{b}$ Substituent groups on boron. 
Kee et al.

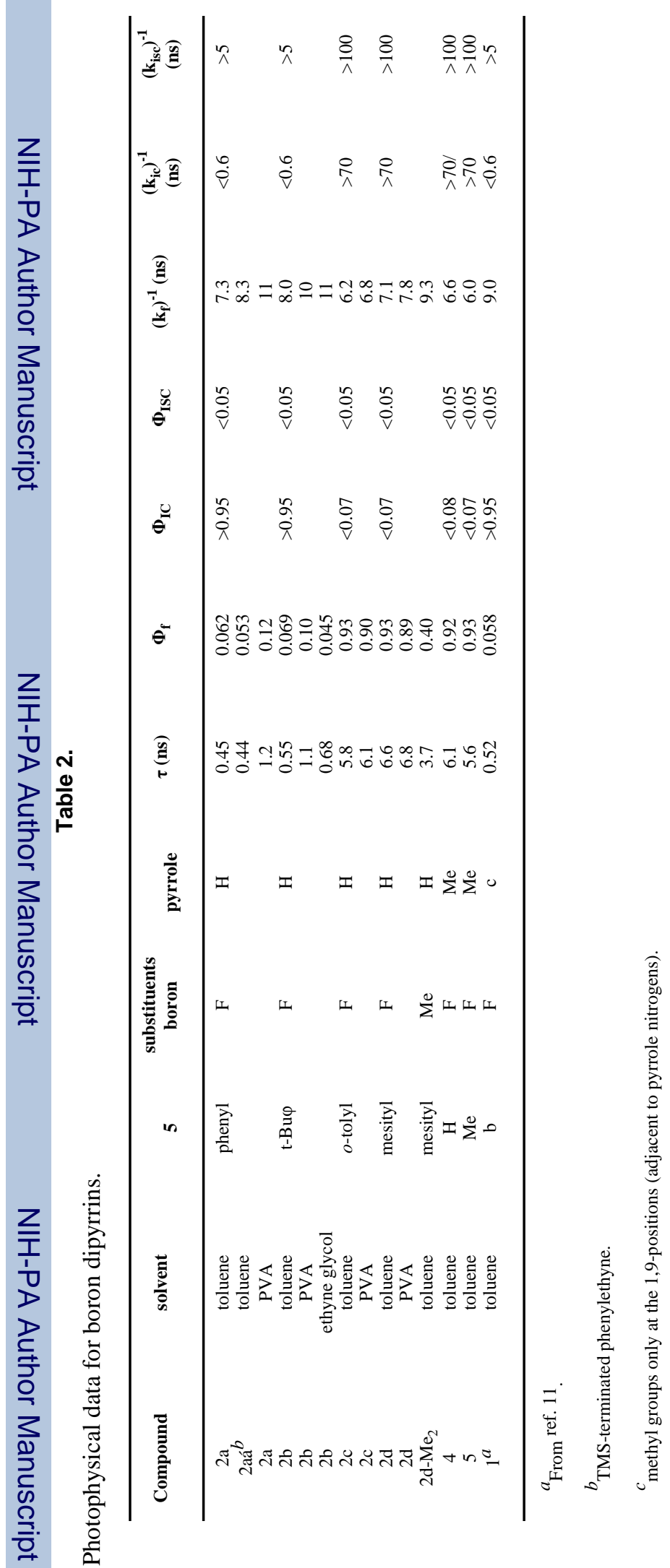


Table 3.

Dominant configurations of boron-dipyrrin states.

\begin{tabular}{ccc}
\hline State & Transition Polarization & Configurations \\
\hline $\mathrm{A}_{1}$ & $\mathrm{z}$ & $\mathrm{b}_{1} \leftarrow \mathrm{b}_{1}$ \\
$\mathrm{~A}_{2}$ & forbidde & $\mathrm{b}_{1} \leftarrow \mathrm{b}_{2}$ \\
$\mathrm{~B}_{1}$ & $\mathrm{x}$ & $\mathrm{b}_{1} \leftarrow \mathrm{a}_{1} ; \mathrm{a}_{2} \leftarrow \mathrm{b}_{2}$ \\
$\mathrm{~B}_{2}$ & $\mathrm{y}$ & $\mathrm{b}_{1} \leftarrow \mathrm{a}_{2}$ \\
\hline
\end{tabular}

